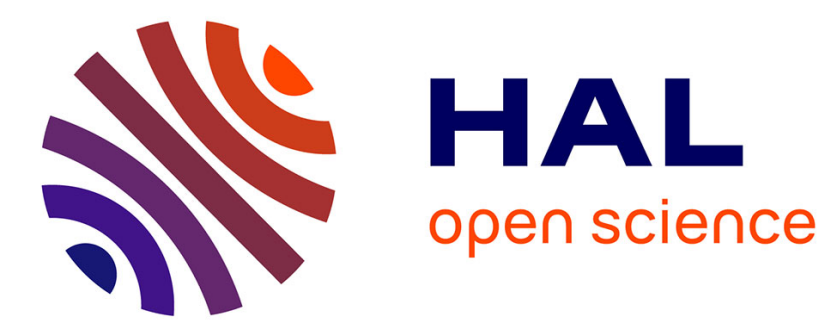

\title{
Wave Analysis in the Atmosphere of Venus Below 100-km Altitude, Simulated by the LMD Venus GCM
}

Sébastien Lebonnois, Norihiko Sugimoto, Gabriella Gilli

\section{To cite this version:}

Sébastien Lebonnois, Norihiko Sugimoto, Gabriella Gilli. Wave Analysis in the Atmosphere of Venus Below 100-km Altitude, Simulated by the LMD Venus GCM. Icarus, 2016, 278, pp.38-51. 10.1016/j.icarus.2016.06.004 . hal-01332089

\section{HAL Id: hal-01332089 \\ https://hal.sorbonne-universite.fr/hal-01332089}

Submitted on 15 Jun 2016

HAL is a multi-disciplinary open access archive for the deposit and dissemination of scientific research documents, whether they are published or not. The documents may come from teaching and research institutions in France or abroad, or from public or private research centers.
L'archive ouverte pluridisciplinaire HAL, est destinée au dépôt et à la diffusion de documents scientifiques de niveau recherche, publiés ou non, émanant des établissements d'enseignement et de recherche français ou étrangers, des laboratoires publics ou privés. 


\title{
Wave Analysis in the Atmosphere of Venus Below 100-km Altitude, Simulated by the LMD Venus GCM
}

\author{
Sébastien Lebonnois ${ }^{\mathrm{a}, *}$, Norihiko Sugimoto $^{\mathrm{b}}$, Gabriella Gilli $^{\mathrm{a}}$ \\ ${ }^{a}$ Laboratoire de Météorologie Dynamique (LMD/IPSL), Sorbonne Universités, UPMC \\ Univ Paris 06, CNRS, 4 place Jussieu, 75252, Paris, France \\ ${ }^{b}$ Department of Physics, Keio Univ. Yokohama, Japan
}

\begin{abstract}
\end{abstract}
A new simulation of Venus atmospheric circulation obtained with the LMD Venus GCM is described and the simulated wave activity is analysed. Agreement with observed features of the temperature structure, static stability and zonal wind field is good, such as the presence of a cold polar collar, diurnal and semi-diurnal tides. At the resolution used (96 longitudes $\times 96$ latitudes), a fully developed superrotation is obtained both when the simulation is initialised from rest and from an atmosphere already in superrotation, though winds are still weak below the clouds (roughly half the observed values). The atmospheric waves play a crucial role in the angular momentum budget of the Venus's atmospheric circulation. In the upper cloud, the vertical angular momentum is transported by the diurnal and semi-diurnal tides. Above the cloud base (approximately 1 bar), equatorward transport of angular momentum is done by polar barotropic and mid- to high-latitude baroclinic waves present in the cloud region, with frequencies between 5 and 20 cycles per

${ }^{*}$ Corresponding author

Email address: Sebastien.Lebonnois@lmd.jussieu.fr (Sébastien Lebonnois) 
Venus day (periods between 6 and 23 Earth days). In the middle cloud, just above the convective layer, a Kelvin type wave (period around $7.3 \mathrm{Ed}$ ) is present at the equator, as well as a low-latitude Rossby-gravity type wave (period around $16 \mathrm{Ed}$ ). Below the clouds, large-scale mid- to high-latitude gravity waves develop and play a significant role in the angular momentum balance.

Keywords: Venus, atmosphere ; Atmospheres, dynamics /, Superrotation ; Numerical modeling

\section{Introduction}

15 angular momentum vertically in the low latitudes was confirmed by recent

The general circulation in Venus's atmosphere is dominated by the phenomenon called superrotation, with most of the atmosphere rotating in the same direction but about sixty times faster than the solid surface. The mechanism that controls this phenomenon combines transport of angular momentum by the mean meridional circulation with compensation done by planetary-scale waves. This idea was originally proposed by Gierasch (1975) and Rossow and Williams (1979), and has been demonstrated in recent years with the study of numerical simulations conducted by General Circulation Models (GCMs) of the Venus's atmosphere (e.g. Yamamoto and Takahashi, 2003; Lee et al., 2007). These tools are very useful to study the wave activity and its role in the angular momentum budget. In addition to the balance between transport by the mean meridional circulation and transport by the horizontal planetary waves, the role of thermal tides that transport works (Takagi and Matsuda, 2007; Lebonnois et al., 2010). The most recent 

${ }_{40}$ 2015), exploring wave solutions and their classification.

41 et al., 2014a,b).

and realistic GCMs either use a full radiative transfer module to compute temperature self-consistently (Lebonnois et al., 2010; Ikeda, 2011; Lee and Richardson, 2011; Mendonca et al., 2015), or force the temperature structuree with carefully prepared heating rate profile and Newtonian cooling (Sugimoto

A variety of waves in Venus's atmosphere has been observed in the cloud region, in the middle cloud from infrared observations or at the cloud-top from reflected visible and ultraviolet sunlight (e.g. Belton et al., 1976; Rossow et al., 1980; Del Genio and Rossow, 1990; Peralta et al., 2008; Piccialli et al., 2014). These observed waves range from planetary-scale waves to small-scale gravity waves. Variability observed in the wind reveals periods from 4-5 Earth days to 1 Venus day, or even longer (Rossow et al., 1990; Kouyama et al., 2013; Khatuntsev et al., 2013) Smahl-scale gravity waves are observed in Venus-Express datasets, with VIRTIS images (Peralta et al., 2008), VMC images (Piccialli et al., 2014), or VeRa radio-occultations (Tellmann et al., 2012). The theoreticat analysis of waves present in Venus's atmosphere is different from the Earth case, because of the cyclostrophic regime and the crucial role played by/the mean zonal wind field. Most analytical works studying Yenus's atmospheric waves use a realistic vertical wind profile, but with a solid-body rotation approximation for the latitudinal wind profile (e.g. Covey and Schubert, 1982; Schinder et al., 1990; Smith et al., 1993). A detailed theoretical analysis of waves in the context of a realistic zonal wind field for Venus has been recently undertaken by Peralta et al. (2014a,b,

Kouyama et al. (2015) explores the possibility of retroactive interactions 

latitude-longitude grid finite-difference dynamical core (e.g. Hourdin et al., ${ }_{64}$ 2006), including a longitudinal polar filter.

between a low-latitude Kelvin type wave and mid-latitude Rossby type waves at the cloud top: the variations of the zonal wind induced by each wave favor the vertical propagation of the other one, that process yields long-term variability of the wind over periods of several Venus days.

Using a high-resolution Venus GCM starting from superrotation and forced by observed heating rate profile and Newtonian cooling, Sugimoto et al. (2014a,b) analysed the wave activity produced in the cloud region. The large vertical zonal wind shear and latitudinal temperature gradient generate the basic state of baroclinic instability in the cloud region. Baroclinic waves develop, and at cloud-top, Rossby type waves are produced by this baroclinic activity. However, further studies of Venus waves using various GCMs are required to get a comprehensive understanding of Venus's atmospheric dynamics.

In this work, we present the recent evolutions of the LMD Venus GCM (Lebonnois et al., 2010), that simulates realistic temperature and zonal wind fields (Section 2), with a detailed analysis of the waves produced in this simulation (Section 3).

\section{Simulations and Validation against Observations}

\subsection{The $L M D$ Venus $G C M$}

The model developed at LMD for the study of Venus's atmosphere has been described in details in Lebonnois et al. (2010). It is based on the LMDZ 
Most of the features of this GCM are similar to those presented in Lebonnois et al. (2010). Among the physical parameterisations, the main difference is the use of a boundary layer scheme taken from Mellor and Yamada (1982) to compute the eddy diffusion coefficient and the time evolution of the mixed variables. The equations used are described in the Appendix B of Hourdin et al. (2002). This boundary layer scheme is based on a more physical representation of the unstable regions. It was successfully used for other planetary applications of the LMD GCM (e.g. Lebonnois et al., 2012a, for Titan). At surface, the drag coefficient is similar to the one used in the previous parameterisation: $C_{d}=\left(0.4 / \ln \left(1+z_{1} / z_{0}\right)\right)^{2}$, where $z_{1}$ is the altitude of the center of the first layer of the model (roughly $10 \mathrm{~m}$ in our case) and $z_{0}$ is the roughness coefficient, taken equal to $1 \mathrm{~cm}$. Otherwise, compared to Lebonnois et al. (2010) we use the same hybrid vertical coordinates with topography (50 vertical levels), the soil model is unchanged, the temperature dependence of the specific heat is taken into account, the radiative transfer includes solar heating rate profiles as a function of solar zenith angle taken from a lookup table based on Crisp (1986), and the infrared net-exchange rate (NER) matrix formulation discussed in Eymet et al. (2009). For the computation of the IR NER matrix, opacity sources (gas, clouds) are taken horizontally uniform and properties are the same as in Lebonnois et al. (2010). The second main difference is the horizontal resolution. For the study that is presented here, the resolution was increased to 96 longitudes by 96 latitudes $\left(3.75^{\circ} \times 1.875^{\circ}\right)$. A few years ago, a simulation was performed with this model with a horizontal resolution of 48 longitudes by 32 latitudes and run for up to 190 Venus days (1 Venus day $(\mathrm{Vd})=117$ Earth days (Ed)) 
starting from an atmosphere already in superrotation. Modeled temperature field was compared with VIRTIS/Venus-Express temperature retrievals to discuss thermal tides above the clouds (Migliorini et al., 2012). It must be noted that when starting from rest, the superrotation was not fully evolved in this version of the GCM, with weaker zonal wind in the deep atmosphere and a peak around $80 \mathrm{~m} / \mathrm{s}$ at the cloud-top, after more than $1000 \mathrm{Vd}$. In the present work, the simulations were started either from rest (for 300 Venus days) or from a zonal wind field already in superrotation (for 190 Venus days). Both simulations converged toward very similar wind fields, meaning that the superrotation is fully evolved by the LMD VGCM from motionless state for the first time. The present work will foeus on the analysis of the simulation started from superrotation.

\section{TABLE 1}

Increasing the resolution had a drawback: it has induced a larger residual term in the angular momentum budget as indicated in Table 1 (that can be compared to simulations presented in Lebonnois et al., 2012b). As mentioned in Lebonnois et al. (2012b), the lack of angular momentum conservation is a problem for simulations of Venus's atmosphere, and Table 1 shows that in this configuration, our GCM has a bias in the angular momentum budget. This is eurrently investigated and needs to be improved in future simulations. This numerical inaccuracy alters the balance of angular momentum transport, the non-zero term due to dynamics in the angular momentum budget being compensated by a non-zero balance of the surface momentum exchanges. However, the convergence between simulations started from rest and started from superrotation gives confidence in the achieved wind dis- 
${ }_{137}$ FIG 3

138 139

FIG 1

\section{FIG 2}

tributions, except near the surface where the winds must be biased. This bias should be taken into account when comparing the resulting simulated circulation to the observations near the surface.

\subsection{Temperature and Winds Fields}

The temperature structure obtained both in the simulation started from superrotation (after $190 \mathrm{Vd}$ ) and in the simulation started from rest (after $300 \mathrm{Vd}$ ) are displayed in Fig. 1. Though the vertical profile is close to the VIRA reference atmosphere (Seiff et al., 1985), the modeled surface temperature is colder than observed, and the temperature in the cloud slightly higher (e.g. Tellmann et al., 2009; Migliorini et al., 2012; Grassi et al., 2014).

The temporal variation of the temperature at a fixed longitude, at cloud top, is shown in Fig. 2. It is dominated by the thermal tides, but highfrequency variations are also clearly present. As in previous simulations (Lebonnois et al., 2010; Mighorini et al., 2012), the semi-diurnal tide is dominant at low latitudes, while the diurnal tide is dominant at high latitudes. The quasi-bidiumal oscillation that was present in L10 is not present in these simulations. This mode of oscillation, which is not understood yet and which is clearly not present in the observations, appeared in previous simulations only when starting from rest, and in simulations when the zonal wind speed in the cloud region remained weaker than observed.

The horizontal structure of the temperature at $3 \times 10^{3} \mathrm{~Pa}$ (roughly $67 \mathrm{~km}$, just below the cloud-top) is shown in Fig. 3, with four panels separated by 

${ }_{163}$ /and strength of the cold collar, since the radiative impact of the clouds in 164

1/100 Vd (1.17 Ed). Though the zonally and temporally averaged latitudinal profiles show a small cold-collar signature at cloud-top, an equator-to-pole contrast around $30 \mathrm{~K}$ is obtained near $60 \mathrm{~km}$ altitude (Fig. 1b). The structure of the cold polar collar is much more visible in the maps shown in Fig. 3 (without zonal and temporal averaging). This structure is very similar to the cold polar collar structure discussed in Ando et al. (2016), which is obtained with the GCM named AFES (Sugimoto et al., 2014a,b). A similar feature was also obtained previously in the works of Lee et al.(2005) and Yamamoto and Takahashi (2012). The model of Lee et al. (2005) obtained quite a different circulation compared to the present result's and to the AFES model, so comparison is difficult. Yamamoto and Takahashi (2012) showed a partially formed cold collar with a warm core, but did not investigate the mechanism producing this feature. In a detailed investigation, Ando et al. (2016) demonstrated the role of the thermal tide in this temperature distribution.

The shape of the polar temperature distribution (cold collar and warm pole) obtained in our results is affected both by the thermal tide and by highfrequency wave activity, though the variations illustrated in Fig. 3 are weak. The contrasts inside the polar regions are also weaker than in the AFES simulations of Ando et al. (2016). Note that AFES is a spectral model used at higher resolution. These two significant differences, spectral model and high resolution should improve the description of the polar region. It must be noted that the latitudinal variation of the cloud structure is not taken into account in any of these works. This could play a role in the shape the polar region is significant, but the results discussed here show that this 
interaction is of second order for the presence of the cold collar.

\section{FIG 4}

The static stability profile is shown in Figure 4. It may be compared to the stability profiles deduced from the Pioneer Venus data (Schubert, 1983), from the Vega 2 entry probe (also reproduced on Fig. 4, Zasova et al., 2007), and from the VeRa/Venus Express data (Tellmann et a1., 2009). The dominant features are the convective region located in the lower and middle clouds (the region with very small stability around $50 \mathrm{~km}$ altitude), the stable layer below the clouds, and the low-stability deep atmosphere (below $30 \mathrm{~km}$ altitude). Compared to the simulations done change of boundary layer scheme has clearly improved the agreement with observations close to the surface. The thickness of the modeled convective region is smaller than in the observations. As mentioned in Lebonnois et al. (2015), the cloud model used to computed the radiative transfer plays a role in this feature. Simulations based on a new cloud model (Haus et al., 2014) in which the thickness of the convective layer is improved compared to observations will be studied in future work.

\section{FIG 5}

FIG 6

The zonally and temporally averaged zonal wind field obtained in the simulation/s presented in Fig. 5, together with the mean meridional circulation. As in Lebonnois et al. (2010), the meridional circulation is divided in three regions along the vertical: a dominant pair of equator-to-pole Hadleylike cells in the lower and middle cloud (roughly $10^{5}$ to $10^{4} \mathrm{~Pa}$ ), a similar pair above $10^{4} \mathrm{~Pa}$ (up to the model top), and another below the clouds, 
though in the deep atmosphere the cell structure is affected by perturbations from topography. The comparison between the modeled zonal wind field and observations has improved compared to Lebonnois et al. (2010) as shown in Fig. 6, where the vertical and latitudinal profiles of the zonal wind are compared to in-situ vertical profiles (adapted from Schubert, 1983) and cloud-tracking latitudinal profiles (taken from Hueso et a1., 2015). The improved treatment of the boundary layer yields more efficient pumping of angular momentum in the deep atmosphere, and thus the zonal wind below the clouds can reach more than $20 \mathrm{~m} / \mathrm{s}$. Although it is approximately half of the observed values (Fig. 6), this is a significant step forward compared to Lebonnois et al. (2010) where the deepest atmosphere was moving with only very weak zonal winds. However, the increased horizontal resolution plays also a role. The simulations previously mentioned, done with the improved boundary layer but at lower horizontal resolution, led to weaker zonal wind (especially in the deep atmosphere) when started from rest. One possible explanation is that the increase in the model grid resolution have improved the representation of the planetary-scale waves at the smallest resolved scales, with therefore an effect on the global angular momentum balance (see in particular Section 3.5 below). This will be investigated in order to confirm the present results.

In the upper region of the clouds, the modeled zonal wind is close to the observations. However, several discrepancies must be mentioned: (1) above the clouds, the zonal wind does not decrease as fast with altitude as the thermal wind computations suggest (e.g. Piccialli et al., 2012) and values around $100 \mathrm{~m} / \mathrm{s}$ extends up to $90 \mathrm{~km}$ altitude; (2) the equatorial jet is too 
strong compared to mid-latitudes - it must be noted that this region is extremely sensitive to radiative forcings, and to details in the model (such as cloud structure), which affect the balance in angular momentum and the zonal wind distribution; (3) the vertical gradient between the lower and upper cloud is higher than observed; (4) the latitudinal gradient in the polar region is too steep compared to the cloud-tracking zonal wind profiles. This is related to the temperature field in the polar vortex (position and amplitude of the cold collar). Several investigations are on-going in order to improve on these discrepancies, mainly regarding the potential role of small-scale processes (gravity waves, convective mixing) and improvements in the radiative transfer. Despite these discrepancies, the overall structure of the zonal wind field is close to the observed structure, and we focus now on the analysis of the waves produced in the GCM simulation.

\section{Wave analysis}

\subsection{Analysis technique}

To study the waves developing in the modeled atmospheric circulation, we use Fast Fourier Transform (FFT) to analyse frequency spectrum of temperature, zonal and meridional wind fields. Modeled atmospheric fields are output with a frequency of 100 points per Venus day. For each grid point in the GCM (given latitude, longitude and pressure level), the FFT is applied to this time series over the last four Venus days of the simulation. The amplitude of the FFT signal at a given frequency is averaged over the longitudes before study. To minimize the impact of the 4 -Vd time window, the time series is multiplied by a triangular window $(0$ at $\mathrm{t}=0$ and $\mathrm{t}=4 \mathrm{Vd}, 2$ at 
$\mathrm{t}=2 \mathrm{Vd})$ (Harris, 1978). Resolution of the FFT spectra is $0.25 / \mathrm{Vd}$, over the range $0.25-50 / \mathrm{Vd}$.

\section{FIG 7}

The amplitude of the FFT of temperature, zonal and meridional wind fields as a function of pressure and frequency are displayed in Fig. 7, for the equator and $70^{\circ} \mathrm{N}$. Several groups of waves appear in these plots:

- The diurnal and semi-diurnal tides are the strongest waves visible above roughly $10^{5} \mathrm{~Pa}$ (see Section 3.3 and Fig. 11 below).

- In the cloud region, between $10^{5} \mathrm{~Pa}$ and $10^{3} \mathrm{~Pa}$, a large group of waves is visible at high latitudes, with frequencies between 5 and $20 / \mathrm{Vd}$.

- At $2 \times 10^{4} \mathrm{~Pa}$, in the equatorial region, a wave is visible around $16 / \mathrm{Vd}$, mostly on the zonal wind. Its harmonics are visible too.

- In the deep atmosphere, below the clouds, waves are mostly visible at high latitudes, with frequencies up to $30 / \mathrm{Vd}$, and a peak near $8 / \mathrm{Vd}$. Additional waves are also visible in the equatorial region, at very low frequencies.

\section{FIG 8}

Latitudinal dependence of the spectra are displayed in Fig. 8 for the cloud region (pressure of $2 \times 10^{4} \mathrm{~Pa}$ ) and for the deep atmosphere (pressure of $1 \times 10^{6} \mathrm{~Pa}$ ). At $2 \times 10^{4} \mathrm{~Pa}$, the diurnal and semi-diurnal tides are visible, as well as the high- to mid-latitude group of waves, with maxima between 10 and $16 / \mathrm{Vd}$. This broad activity from mid- to high-latitudes, without a typical frequency, is certainly resulting from instability (barotropic or baroclinic) 
in this region (see Section 3.4). Near 16/Vd, the equatorial wave mentioned previously is also visible in the zonal wind (with its harmonics) and temperature. In the meridional wind, this wave is seen at low latitudes but not on the Equator. These are characteristics of a Kelvin type wave (as confirmed by its horizontal structure, see Section 3.4 below). Another low-latitude wave is seen at a frequency around $8 / \mathrm{Vd}$, with the opposite characteristics: visible only in the meridional wind at the equator, and in the zonal wind at low latitudes. This could indicate a Rossby-gravity type wave. At $1 \times 10^{6} \mathrm{~Pa}$, the distribution of the waves in the mid- to high-latitudes is displayed.

It is also possible to apply the FFT bi-dimensionally (longitude-time) for a given latitude and pressure level. In this case, the direction of the wave propagation and the longitudinal wavenumber are obtained. Note that the mean zonal flow is westward, as Venus's/surface is in retrograde rotation compared to the Earth's rotation. Apart from the thermal tides that are eastward (along with the Sun), all the previously mentioned waves are westward, with wavenumber 1 for the group in the clouds and wavenumber 2 or 3 for the group in the deep atmosphere. In the cloud, the Kelvin type wave is moving almost at the same speed as the mean zonal flow, while its harmonics are faster. The other group is slower, thus propagating eastward relatively to the mean flow. In the deep atmosphere, waves both faster and slower than the zonal wind are present.

\section{FIG 9}

To separate the different waves, we apply filters on the FFT spectra, before applying a reverse FFT. At a given frequency $f_{0}$, the shape of the lowpass filter is: 1 for $f<f_{0}-\delta f, 0$ for $f>f_{0}+\delta f$ and $0.5+\sin (\pi / 2 \times$ 
$\left.\left(f_{0}-f\right) / \delta f\right) / 2$ in between, where $\delta f$ is $1 / \mathrm{Vd}$. A similar shape is applied for a highpass filter, and a bandpass filter is obtained between two frequencies $f_{1}$ and $f_{2}$ by combining a highpass filter at $f_{1}$ and a lowpass filter at $f_{2}$. Filtering is done at these cutting frequencies: 4, 10 and $22 / \mathrm{Vd}$, as illustrated in Fig. 9.

\subsection{Angular momentum transport}

The specific angular momentum $M$ is computed as $M=u \times d \cos \phi$, where $a$ is Venus's radius, $\phi$ is the latitude, and $u$ is the zonal wind. The total meridional transport $[\overline{v M}]$ of the angular momentum can be decomposed as:

$$
[\overline{v M}]=[\bar{v}][\bar{M}]+\left[\overline{v^{*}}\right]\left[\overline{M^{*}}\right] \nsucc\left[\overline{v^{\prime} M^{\prime}}\right],
$$

where $[M]$ is the zonal mean, $\bar{M}$ the temporal average, $M^{*}=M-[M]$, and $M^{\prime}=M-\bar{M} \cdot[\bar{v}][\bar{M}]$ is the contribution from the mean meridional circulation $(\mathrm{MMC}),\left[\overline{v^{*}}\right]\left[\overline{\left.M^{*}\right]}\right.$ is the contribution from stationary waves, and $\left[\overline{v^{\prime} M^{\prime}}\right]$ is the contribution from transients waves. The same equation applies for vertical transport of $M$, using the vertical wind $w$ (in $\mathrm{Pa} / \mathrm{s}$ ) instead of the meridional wind $v$. The stationary waves component appears to be negligible.

The transient term can be computed either with the complete perturbation field $M^{\prime}$, or with the perturbations obtained after wave filtering. This allows to separate the contribution of each group of waves in the horizontal or vertical transport.

\section{FIG 10}

The total horizontal transport is obtained with a vertical integration of each term, weighted by the mass of each cell. The total vertical transport is 
done with a horizontal integration, weighted only by $\cos \phi$. Figure 10 shows these total horizontal and vertical transports of angular momentum. In the horizontal, the poleward MMC transport is compensated by the equatorward transport by the waves. Note that this plot is similar if the vertical integration is done only down to the cloud bottom, which means that this balance is obtained both in the cloud region and in the deep atmosphere. In the vertical, the upward MMC transport is compensated by downward transport by the waves. In the cloud region and above, this transport is mostly done by the thermal tides. This is consistent with our previous analysis done in Lebonnois et al. (2010). Therefore, in our simulations, superrotation is obtained and maintained through the Gierasch-Rossow-Williams mechanism (mean meridional circulation and horizontal waves), with a significant additional contribution of the thermal tides in the global angular momentum balance. It must be noted that the residual poleward transport is a combination of the latitudinal distribution of the numerical inaccuracies discussed at the end of Section 2.1, and of latitudinal redistribution of angular momentum at the surface. In the vertical, the net transport should be zero and the residual is also an indication of these numerical inaccuracies.

\subsection{Thermat Tides}

\section{FIG 11}

Latitude vs pressure maps of the FFT spectrum of the temperature, for the frequencies $1 / \mathrm{Vd}$ and $2 / \mathrm{Vd}$ are plotted in Fig. 11 . They show that the tides are mostly visible above $10^{4} \mathrm{~Pa}$. The semi-diurnal tide is dominating in the low to mid latitudes between $10^{4} \mathrm{~Pa}$ and $10^{2} \mathrm{~Pa}$, while the diurnal tide dominates above $10^{2} \mathrm{~Pa}$, and at high latitudes. This is consistent with the 
analysis of our previous simulations at lower resolution that was discussed in Migliorini et al. (2012), in comparison with VIRTIS/Venus-Express dataset analysis. The amplitude of the diurnal and semi-diurnal tides ( 5 to $10 \mathrm{~K}$ in the pressure range $10^{4}$ to $10^{2} \mathrm{~Pa}$ ) is consistent with the observed values (Migliorini et al., 2012; Grassi et al., 2014).

\subsection{Waves in the Cloud Layer and Above}

\section{FIG 12}

To illustrate the distribution of the waves present in the cloud region and above, Fig. 12 shows the latitude vs pressure maps of the FFT spectra of the zonal and meridional winds for two frequencies, $7.25 / \mathrm{Vd}$ and $16 / \mathrm{Vd}$. One group is located on the poles, where the latitudinal gradient of the zonal wind speed is large, so the waves would be caused by barotropic instability. Another group is at mid latitudes (40) to $70^{\circ}$ ), in a region dominated by a large vertical gradient of the zonal wind. The level at which the phase speed of these disturbances is equal to the background speed (steering level) is deeper for the lower frequency waves, which explains that this mid- to high-latitude wave group is located deeper $\left(5 \times 10^{4}\right.$ to $\left.1 \times 10^{5} \mathrm{~Pa}\right)$ than the higher frequency wave group (around $2 \times 10^{4} \mathrm{~Pa}$ ). On the equatorial region, the Kelvin type and mixed Rossby-gravity type waves are also visible.

As seen in Fig. 1, the latitudinal gradient between the equator and the poles at $1 \times 10^{4} \mathrm{~Pa}$ (roughly $60 \mathrm{~km}$ altitude) is around $30 \mathrm{~K}$. This latitudinal gradient of temperature and the strong vertical zonal wind shear in the $10^{5}$ $10^{4} \mathrm{~Pa}$ region may be the source of baroclinic instabilities. A necessary condition for baroclinic instability has been used by Sugimoto et al. (2014a), following Young et al. (1984), though the validity of this criterion for Venus 
may be taken with caution. The following latitudinal gradient must change sign for instabilities to occur:

$$
\frac{\partial \bar{q}}{\partial \phi}=2 a \Omega_{u} \cos \phi-\frac{\partial}{\partial \phi}\left(\frac{1}{\cos \phi} \frac{\partial}{\partial \phi}(\cos \phi \tilde{u})\right)-\frac{4 a^{2} \Omega_{u}^{2} \sin ^{2} \phi}{p} \frac{\partial}{\partial z}\left(\frac{p}{N^{2}} \frac{\partial \tilde{u}}{\partial z}\right)
$$

where $\Omega_{u}$ includes the rotation of the atmosphere at a reference level of $3 \times 10^{4} \mathrm{~Pa}$, and $\tilde{u}$ is the mean zonal field relative to this referencelevel. $N^{2}$ is the Brunt-Väisälä frequency, computed from the temperature lapse rate:

$$
N^{2}=\frac{g}{T}\left(\frac{\partial T}{\partial z}+\frac{g}{c_{p}}\right) .
$$

Such a criterion appears to be fulfilled in the mid to high latitudes, between $10^{5}-10^{4} \mathrm{~Pa}$, as seen in Fig. $12 \mathrm{c}$, Note also that criteria are also satisfied in the polar region because of the second term in Eq.2, suggesting that barotropic instability would happen.

\section{FIG 13}

Figure 13a shows the vertical and longitudinal structure of the waves at $45^{\circ} \mathrm{N}$, for temperature and meridional wind fields filtered in the $[10-22] / \mathrm{Vd}$ frequency range. Both present a tilt from up-east to down-west. They are phase-shifted by approximately a quarter period. This structure is similar to the one obtained in the GCM simulations of Sugimoto et al. (2014b) (their Fig. 2; note that in their simulations, the zonal wind is flowing from west to east while it is flowing from east to west in our plots) and it is characteristic of a baroclinic mode. At $80^{\circ} \mathrm{N}$ (Fig. 13b), the structure is different and the wave activity is barotropic, rather than baroclinic. These mid-latitude baroclinic activity may be related to the Rossby waves obtained 
at mid-latitude in the analysis of cloud-tracking observations by Del Genio and Rossow (1990) (Pioneer Venus UV images) and by Kouyama et al. (2013) (VMC/Venus Express images). However, due to the model-dependence of the wave and mean-flow interactions, further investigations are needed to assess the robustness of possible correlations with observed waves.

\section{FIG 14}

The latitudinal transport of heat $\overline{\left(C_{p} T\right)^{\prime} v^{\prime}}$ in the cloud region is plotted in Fig. 14a for filtered fields in the frequency range [4-10]/Vd, and Fig. 14b for filtered fields in the frequency range [10-22]/Vd. In the region where they develop (30 to $70^{\circ}$ of latitude, pressures around $2 \times 10^{4} \mathrm{~Pa}$ for frequency range [10-22]/Vd and deeper, around $5 \times 10^{4}$ to $1 \times 10^{5} \mathrm{~Pa}$ for frequency range [4$10] / \mathrm{Vd})$, the baroclinic waves transport heat poleward. Below, equatorward transport is visible, so that these waves tend to transport heat as in a direct meridional cell, a behavior that was already mentioned in Sugimoto et al. (2014b).

The latitudinal transport of angular momentum by these waves is shown in Fig. 14c,d. Equatorward transport is dominant in the frequency range [10$22] / \mathrm{Vd}$ around $3 \times 10^{4} \mathrm{~Pa}$, but is also noted near $1 \times 10^{5} \mathrm{~Pa}$ for frequency range [4-10]/Vd/ In the region around $60^{\circ}$ and $5 \times 10^{4} \mathrm{~Pa}$, poleward transport of angular momentum by the waves is visible, which can be related to the local jet in this region (as seen in Fig. 5). In the equatorial region around $2 \times 10^{4} \mathrm{~Pa}$, the Kelvin like wave transfers both heat and momentum equatorward.

\section{FIG 15}

In the equatorial region, at $2 \times 10^{4} \mathrm{~Pa}$, the horizontal structure of the two low-latitude waves previously mentioned, with frequencies around 7.25 and 
${ }_{427}$ a conclusion consistent with the works of Del Genio and Rossow (1990) and 428

16/Vd, are shown in Fig. 15. Both waves are westward, with a wavenumber 1. The typical structure of a Kelvin type wave is visible in Fig. 15b (frequency $\sim 16 / \mathrm{Vd}$ ). This Kelvin type wave is propagating faster than the zonal wind, as seen on Fig. 8(c,e), except at the equator where it accelerates the flow. The structure of the other wave (Fig. 15a) has similar characterístics as a Rossby-gravity type wave of order $n=0$ as in Fig. 6a of Matsuno (1966). It is propagating slower than the zonal wind. The pressure at which these two waves develop $\left(2 \times 10^{4} \mathrm{~Pa}\right)$ corresponds to a layer where the stability becomes large, just above the convective region in the cloud.

Both type of waves are suggested in the analysis of cloud-top observations by Del Genio and Rossow (1990) from Pioneer Venus UV images and by Kouyama et al. (2013) from VMC/Venus Express images. In Del Genio and Rossow (1990), the Kelvin wave is present when the zonal flow is faster. Kouyama et al. (2013) have a different conclusion with their analysis: the Kelvin wave appears when the zonal wind is slower, while Rossby waves prevail when the zonal wind is faster. However, it must be noted that in our work, these waves appear in the middle cloud and do not propagate upward to the cloud-top region. The vertical shape of the equatorial zonal wind field and its time variations are crucial to the development and propagation of these waves, and they are therefore very model-dependent. In the simulation started from rest, the Kelvin type wave was not present after $200 \mathrm{Vd}$, but has developed after $300 \mathrm{Vd}$ when the equatorial jet is stronger. This suggests that wave and mean-flow interaction may be significant in the equatorial region, Kouyama et al. (2013). Time-variation and interaction of this wave with the 


$$
451
$$

mean flow will be the focus of a future more detailed study.

\subsection{Waves Below the Clouds}

\section{FIG 16}

In the deep atmosphere, the dominant waves are seen at mid to highlatitudes, with frequencies up to $30 / \mathrm{Vd}$, as seen in Fig. 8. The dominant frequency in the spectra is around 7-8/Vd. Fig. 16 shows the contributions of these waves in the angular momentum transport. They transport momentum downward and equatorward in the mid- to high-latitude regions of the deep atmosphere. This is the dominant contribution for transients, that balances the horizontal and vertical transport of angular momentum by the mean meridional circulation below the coulds.

\section{FIG 17}

\section{FIG 18}

Figure 17 illustrates the meridional distribution of these waves. Meridional wind perturbations are associated to temperature perturbations: when the meridional wind converges, the temperature increases, and reversely, negative temperature perturbations are associated to region where the meridional wind diverges. This is characteristic of gravity waves (e.g. Holton, 2004). Wave trains are visible in Fig. 18, with wavenumbers 2 to 3 . The wind field presents strong convergence and divergence, but rather low vorticity, which is again in favor of gravity waves. These waves are therefore identified as gravity waves. The source region is the stable zone below the clouds (around 2-3 $\times 10^{5} \mathrm{~Pa}$ ), where these waves may be excited through the perturbations in temperature induced in this layer by the cloud-region baroclinic waves. Note that these waves are completely different from the small-scale 
475

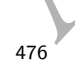

gravity waves that are observed at and above the cloud-top region in the images and radio-occultation data from Venus-Express, which are supposed to be generated in the convective region located in the middle cloud and propagate upwards. Small-scale gravity waves generated by this convection and propagating downwards may also be present in the deep atmosphere, but they can not be resolved by the GCM and need to be parameterized, which is not done in this work.

\section{FIG 19}

Figure 19 shows the temporal evolution of these waves both vertically and horizontally. Propagation is downward and equatorward, inducing momentum transport where the mean zonal wind is smaller than in their source region. These large-scale downward-propagating gravity waves have never been suggested before. They were not present in the LMD Venus GCM simulations done at lower resolution. The increase in horizontal resolution may have improved the representation of wave activity at the lowest resolved scale, and favored the development of these gravity waves. Since their role in the angular momentum budget is significant in the deep atmosphere in the present simulations, their presence needs to be confirmed in future works, as well as their role in the superrotation of the atmosphere below the clouds.

\section{Conclusion}

The LMD Venus GCM was used in this work to produce an updated simulation of Venus atmospheric circulation. At the resolution used here (96 longitudes $\times 96$ latitudes), convergence is obtained when started from rest or from an atmosphere already in superrotation, though numerical inaccu- 
racies in the dynamical core have increased with resolution and affect the angular momentum budget. The temperature structure features a vertical profile close to observations, yet slightly colder in the deep atmosphere, a vertical profile of static stability in agreement with observations, dominating thermal tides above the cloud-base, and a cold polar collar structure in the upper cloud. To improve the fit of temperatures to observations, future work will include improvement in the radiative transfer properties (based on our recent work Lebonnois et al., 2015), and taking into account the latitudinal variations of the cloud. The modeled zonal wind distribution presents a fully developed superrotation, though winds are still weak below the clouds (roughly half the observed values).

We have analysed the waves present in this simulation, and their role in the balance of angular momentum budget in the atmosphere of Venus. The role of diurnal and semi-diurnal tides in vertical angular momentum transport is confirmed in the upper cloud. Polar barotropic and mid- to high-latitude baroclinic waves are present in the cloud region, with frequencies between 5 and 20 cycles per Venus day (periods between 6 and 23 Earth days), that redistribute angular mómentum significantly. In the middle cloud, just above the convective layer, a Kelvin type wave (period around 7.3 Ed) is present at the equator, as well as a low-latitude Rossby-gravity type wave (period around $16 \mathrm{Ed}$ ), but the characteristics of these wave activities and their interaction with the background zonal wind may be very sensitive to the modeled circulation and comparison with cloud-top observations should be done with caution. Below the clouds, wave activity that transport angular momentum both downward and equatorward is dominated by large-scale 
D. ${ }_{525} \mathrm{n}^{\circ} 11167$.

mid- to high-latitude gravity waves. The presence and role of these waves was never mentioned in previous works. However, their sensitivity to details of the model needs to be assessed more completely, so that their role in the superrotation mechanism on Venus may be robustly established.

To assess the robustness of the wave activity in the different atmospheric region of Venus's atmosphere and their role in the mechanisms of superrotation, further investigations should always explore how sensitive these different waves are to the background circulation and temperature structure, and to the model and configuration used. In particular, the numerical bias in the angular momentum budget seen in the present simulations might affect the wave activity, and this analysis will need to be confirmed by using a more conservative dynamical core. However, the waves analysed in the present work present many similarities with the ones developing in the AFES Venus GCM (Sugimoto et al., 2014a,b; Ando et al., 2016), though forcing conditions are very different between these two GCMs. This brings confidence in the robustness of these features and of their role in the mechanism of superrotation. Temporal variability over long timescales (several Earth years, tens of Venus days) needs to be investigated too, since hints of such variability are present in the analysis of observational datasets.

\section{Acknowledgements}

This work was supported by the Centre National d'Etudes Spatiales (CNES). GCM simulations were done at CINES, France, under the project 


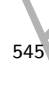

544 Grassi, D., Politi, R., Ignatiev, N. I., Plainaki, C., Lebonnois, S., Wolken-

\section{References}

Ando, H., Sugimoto, N., Takagi, M., Kashimura, H., Imamura, T., Matsuda, Y., 2016. The puzzling Venusian polar atmospheric structure reproduced by a general circulation model. Nature Comm. 7, 10398.

Belton, M. J. S., Smith, G. R., Schubert, G., del Genio, A. D., 1976. Cloud patterns, waves and convection in the Venus atmosphere. J. Atm. Sci. 33, 1394-1417.

Covey, C., Schubert, G., 1982. Planetary-scale waves in the Venus atmosphere. J. Atm. Sci. 39, 2397-2413.

Crisp, D., 1986. Radiative forcing of the Venus mesosphere. I - Solar fluxes and heating rates. Icarus $67,484-514$.

Del Genio, A. D., Rossow, W. B., 1990. Planetary-scale waves and the cyclic nature of cloud top dynamics on Venus. J. Atmos. Sci. 47, 293-318.

Eymet, V., Fournier, R., Dufresne, J.-L., Lebonnois, S., Hourdin, F., Bullock, M. A., 2009. Net-exchange parameterization of the thermal infrared radiative transfer in Venus' atmosphere. J. Geophys. Res. 114, E11008.

Gierasch, P., 1975. Meridional circulation and the maintenance of the Venus atmospheric rotation. J. Atmos. Sci. 32, 1038-1044. berg, P., Monatbone, L., Migliorini, A., Piccioni, G., Drossart, P., 2014. The Venus nighttime atmosphere as observed by VIRTIS-M instrument. 
Ikeda, K., 2011. Development of Radiative Transfer Model for Venus Atmosphere and Simulation of Superrotation Using a General Circulation Model. Ph.D. thesis, University of Tokyo.

Khatuntsev, I. V., Patsaeva, M. V., Titov, D. V., Ignatiev, N. I., Turin, A. V., Limaye, S. S., Markiewicz, W. J., Almeida, M., Roatsch, T., Moissl, R., 2013. Cloud level winds from the Venus Express Monitoring Camera imaging. Icarus 226, 140-158.

Kouyama, T., Imamura, T., Nakamura, M., Satoh, T., Futaana, Y., 2013. Long-term variation in the cloud-tracked zonal velocities at the cloud top of Venus deduced from Venus Express VMC images. J. Geophys. Res. Planets $118,37-46$.

Kouyama, T., Imamura, T., Nakamura, M., Satoh, T., Futaana, Y., 2015. Vertical propagation of planetary-scale waves in variable background winds in the upper cloud region of Venus. Icarus 248, 560-568.

Lebonnois, S., Burgalat, J, Rannou, P., Charnay, B., 2012a. Titan Global Climate Model: new 3-dimensional version of the IPSL Titan GCM. Icarus $218,707-722$

Lebonnois, S., Covey, C., Grossman, A., Parish, H., Schubert, G., Walterscheid, R., Lauritzen, P., Jablonowski, C., 2012b. Angular momentum budget in General Circulation Models of superrotating atmospheres: A critical diagnostic. J. Geophys. Res. 117, E12004.

Lebonnois, S., Eymet, V., Lee, C., Vatant d'Ollone, J., 2015. Analysis of 


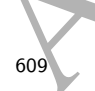

610

the radiative budget of Venus atmosphere based on infrared Net Exchange Rate formalism. J. Geophys. Res. Planets 120, 1186-1200.

Lebonnois, S., Hourdin, F., Eymet, V., Crespin, A., Fournier, R., Forget, F., 2010. Superrotation of Venus' atmosphere analysed with a full General Circulation Model. J. Geophys. Res. 115, E06006.

Lee, C., Lewis, S. R., Read, P. L., 2005. A numerical model of the atmosphere of Venus. Adv. Space Res. 36, 2142-2145.

Lee, C., Lewis, S. R., Read, P. L., 2007. Superrotation in a Venus general circulation model. J. Geophys. Res. 112, E04S11.

Lee, C., Richardson, M. I., 2011. Realistic Solar and Infra-Red Radiative Forcing within a Venus GCM. AGU Fall Meeting Abstracts, A1642.

Matsuno, T., 1966. Quasi-geostrophic motions in the equatorial area. J. of Met. Soc. Japan 44, 25-43.

Mellor, G. L., Yamada, T. 1982. Development of a turbulent closure model for geophysical fluid problems. Rev. Geophys. Space Phys. 20, 851-875.

Mendonca, J M., Read, P. L., Wilson, C. F., Lee, C., 2015. A new fast and flexible radiatif transfer method for Venus general circulation models. Planet. \& Space Sci. 105, 80-93.

Migliorini, A., Grassi, D., Montabone, L., Lebonnois, S., Drossart, P., Piccioni, G., 2012. Investigation of air temperature on the nightside of Venus derived from VIRTIS-H on board Venus-Express. Icarus 217, 640-647. 
611

Peralta, J., Hueso, R., Sanchez-Lavega, A., Piccioni, G., Lanciano, O., Drossart, P., 2008. Characterization of mesoscale gravity waves in the upper and lower clouds of Venus from VEX-VIRTIS images. J. Geophys. Res. 113, E00B18.

Peralta, J., Imamura, T., Read, P. L., Luz, D., Piccialli, A., López-Valverde, M. A., 2014a. Analytical Solution for Waves in Planets with Atmospheric Superrotation. I. Acoustic and Inertia-Gravity Waves. Astrophys. J. Suppl. $213,17$.

Peralta, J., Imamura, T., Read, P. L., Luz, D., Piccialli, A., López-Valverde, M. A., 2014b. Analytical Solution for Waves in Planets with Atmospheric Superrotation. II. Lamb, Surface, and Centrifugal Waves. Astrophys. J. Suppl. 213, 18.

Peralta, J., Sánchez-Lavega, A., López-Valverde, M. A., Luz, D., Machado, P., 2015. Venus's major cloud feature as an equatorially trapped wave distorted by the wind, Geophys. Res. Lett. 42, 705-711.

Piccialli, A., Tellmann, S., Titov, D. V., Limaye, S. S., Khatuntsev, I. V., Pätzold, M., Häusler, B., 2012. Dynamical properties of the Venus mesosphere from the radio-occultation experiment VeRa onboard Venus Express. Icarus 217, 669-681.

Piccialli, A., Titov, D. V., Sanchez-Lavega, A., Peralta, J., Shalygina, O., Markiewicz, W. J., Svedhem, H., 2014. High latitude gravity waves at the Venus cloud tops as observed by the Venus Monitoring Camera on board Venus Express. Icarus 227, 94-111. 
634

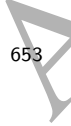

Rossow, W. B., del Genio, A. D., Eichler, T., 1990. Cloud-tracked winds from Pioneer Venus OCPP images. J. Atm. Sci. 47, 2053-2084.

Rossow, W. B., del Genio, A. D., Limaye, S. S., Travis, L. D., 1980. Cloud morphology and motions from Pioneer Venus images. J. Geophys.Res. 85, 8107-8128.

Rossow, W. B., Williams, G. P., 1979. Large-scale motion in the Venus' stratosphere. J. Atmos. Sci. 36, 377-389.

Schinder, P. J., Gierasch, P. J., Leroy, S. S., Smith, M. D., 1990. Waves, advection, and cloud patterns on Venus. J. Atm. Sci. 47, 2037-2052.

Schubert, G., 1983. General circulation and the dynamical state of the Venus atmosphere. In: D. M. Hunten, L. Colin, T. M. Donahue and V. I. Moroz (Ed.), Venus. Univ. of Arizona Press, pp. 681-765.

Seiff, A., Schofield, J. T., Kliore, A. J., et al., 1985. Model of the structure of the atmosphere of Venus from surface to $100 \mathrm{~km}$ altitude. Adv. Space Res. 5 (11), 3-58.

Smith, M. D., Gierasch, P. J., Schinder, P. J., 1993. Global-scale waves in the Venus atmosphere. J. Atm. Sci. 50 (24), 4080-4096.

Sugimoto, N., Takagi, M., Matsuda, Y., 2014a. Baroclinic instability in the Venus atmosphere simulated by GCM. J. Geophys. Res. Planets 119, 19501968.

Sugimoto, N., Takagi, M., Matsuda, Y., 2014b. Waves in a Venus general circulation model. Geophys. Res. Lett. 41, 7461-7467. 
656

Takagi, M., Matsuda, Y., 2007. Effects of thermal tides on the Venus atmospheric superrotation. J. Geophys. Res. 112, D09112.

Tellmann, S., Häusler, B., Hinson, D. P., Tyler, G. L., Andert, T. P., Bird, M. K., Imamura, T., Pätzold, M., Remus, S., 2012. Small-scale temperature fluctuations seen by the VeRa Radio Science Experiment on Venus Express. Icarus 221, 471-480.

Tellmann, S., Pätzold, M., Hausler, B., Bird, M. K., Tyler, G. L., 2009. Structure of the Venus neutral atmosphere as observed by the radio science experiment VeRa on Venus Express. J. Geophys. Res. 114, E00B36.

Yamamoto, M., Takahashi, M., 2003. The Fully Developed Superrotation Simulated by a General Circulation Model of a Venus-like Atmosphere. J. Atm. Sc. 60, 561-574.

Yamamoto, M., Takahashi, M., 2012. Venusian middle-atmospheric dynamics in the presence of a strong planetary-scale 5.5-day wave. Icarus 217, $702-$ 713.

Young, R. E., Pfister, L., Houben, H., 1984. Baroclinic instability in the Venus atmosphere. J. Atmos. Sci. 41, 2310-2333.

Zasova, L. N., Ignatiev, N. I., Khatuntsev, I. A., Linkin, V., 2007. Structure of the Venus atmosphere. Planet. \& Space Sci. 55, 1712-1728. 
Table 1: Values of the terms in the total angular momentum budget, averaged over the last $2 \mathrm{Vd}$ (units are $10^{18} \mathrm{~kg} \mathrm{~m}^{2} \mathrm{~s}^{-2}$ ).

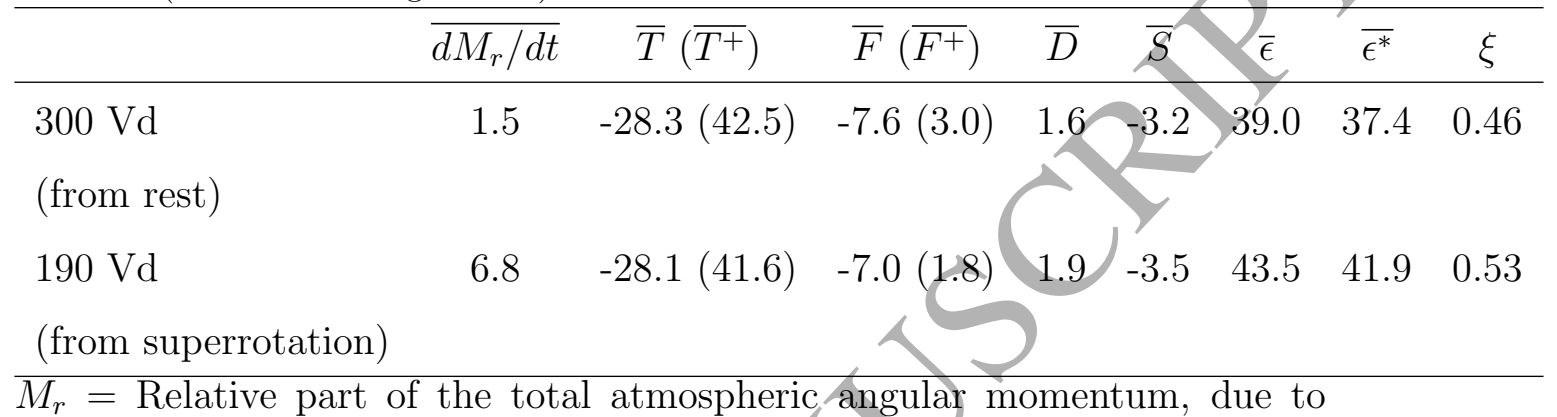

zonal wind $u$

$T=$ Mountain torque on the atmosphere due to topography $\left(T^{+}\right.$is its positive (source) component)

$F=$ Surface torque on the atmosphere due to friction $\left(F^{+}\right.$is its positive (source) component)

$D=$ Residual torque due to conservation errors in the horizontal dissipation parameterization

$S=$ Torque on the atmosphere due to upper boundary conditions (sponge layer)

$\epsilon=$ Residual numerical rate of total angular momentum variation due to conservation errors in the dynamical core $\epsilon^{*}=S+D+\epsilon$, should theoretically be zero

$\xi=$ Ratio between $\left|\overline{\epsilon^{*}}\right|$ and $\operatorname{Max}\left(\overline{T^{+}}+\overline{F^{+}},\left|\overline{T^{-}}+\overline{F^{-}}\right|\right.$) (Lebonnois et al., 2012b) 
a)

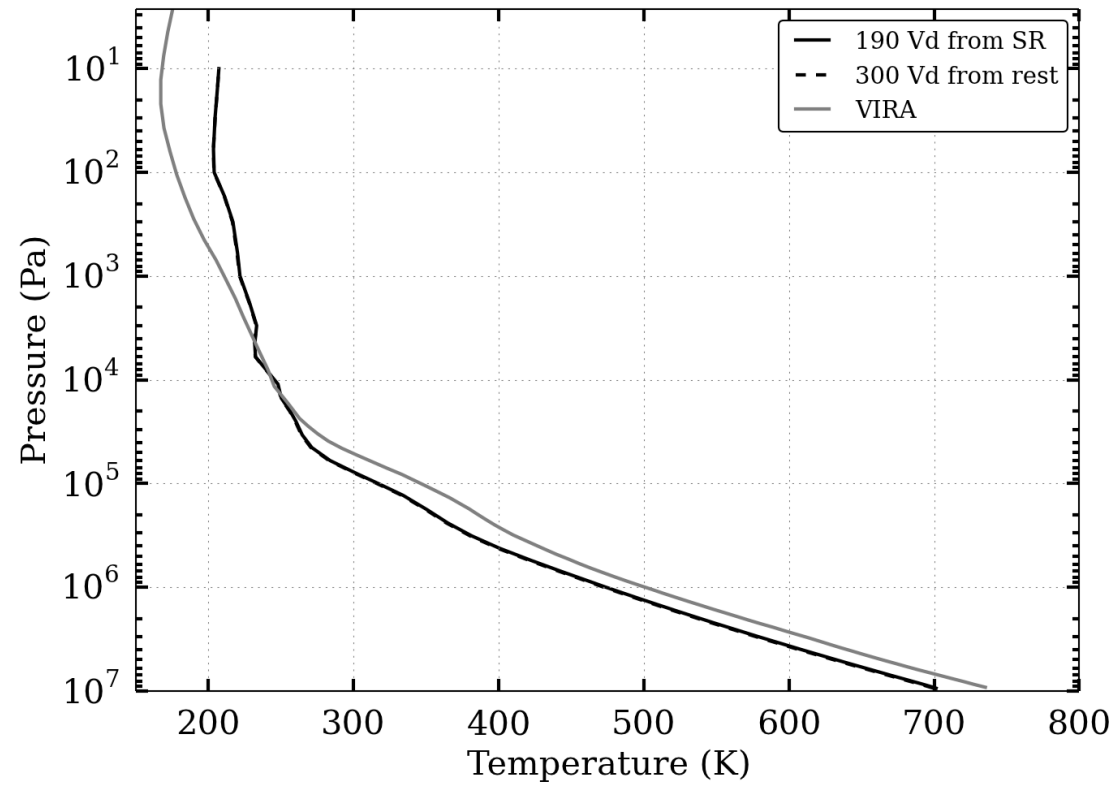

b)

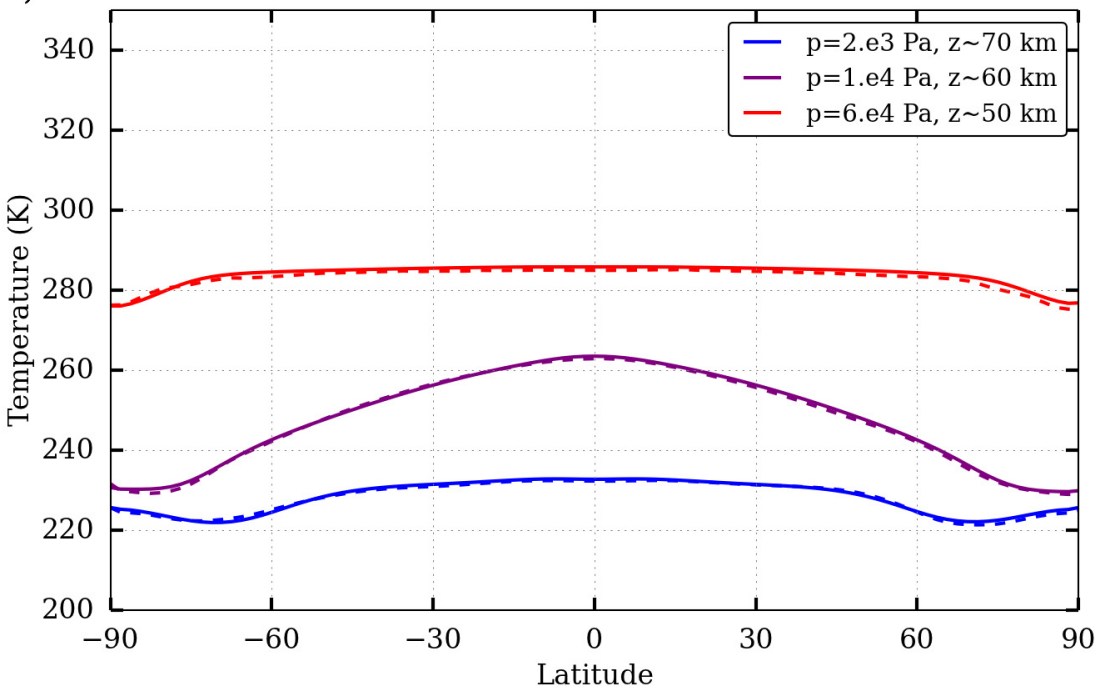

Figure 1: Temperature structure obtained in the simulation started from superrotation, after 190 Vdays: (a) vertical profile of globally averaged temperature; (b) latitudinal profiles of temperature (zonal and temporal average) at roughly 50, 60 and $70 \mathrm{~km}$ altitude. The dashed lines are from the simulation st33ted from rest, after $300 \mathrm{Vd}$. 


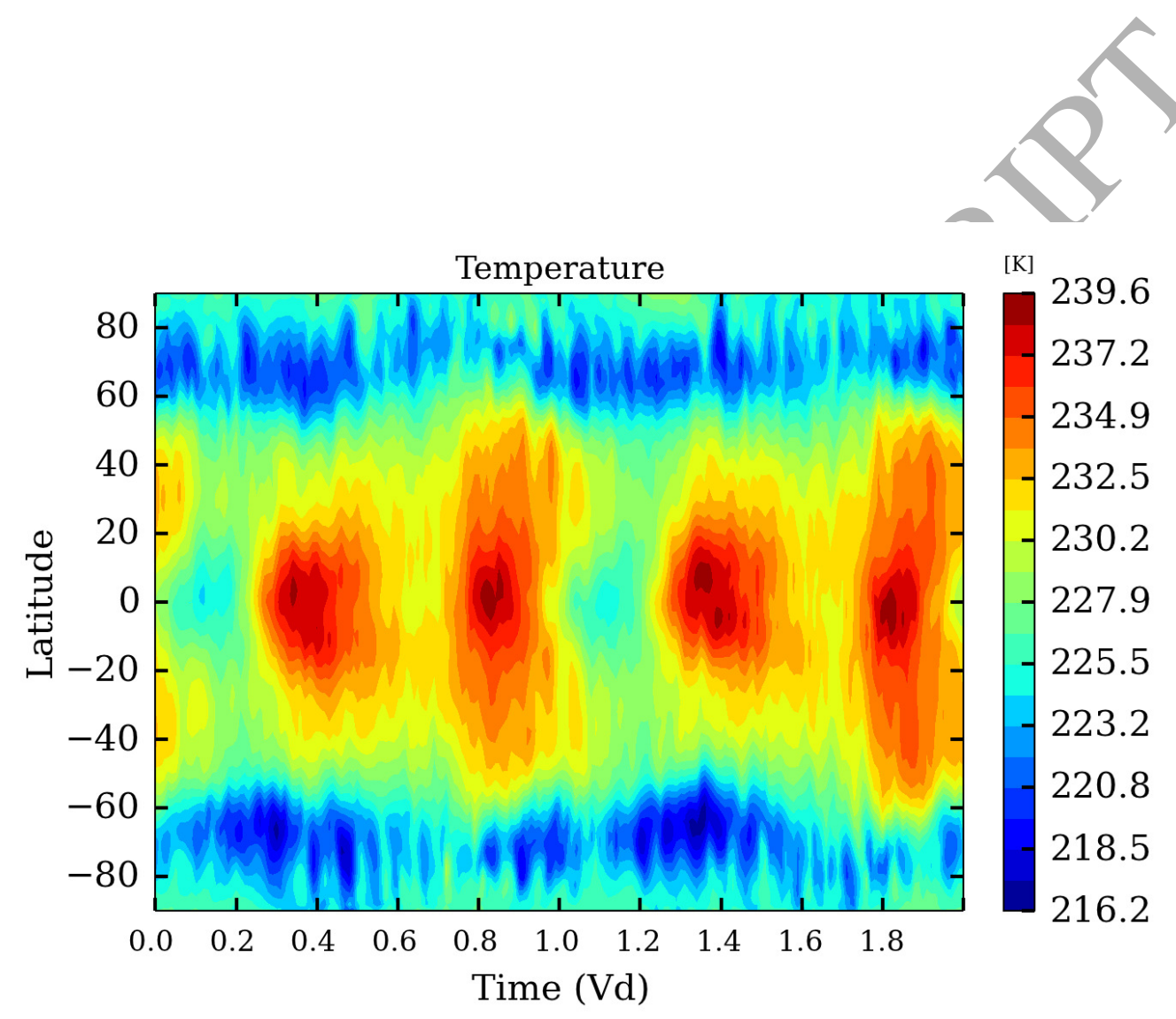

Figure 2: Temporal variations of the temperature field at pressure $\mathrm{p}=2 \times 10^{3} \mathrm{~Pa}$ (near $70 \mathrm{~km}$ altitude, cloud top), and longitude 0 , for the last 2 Venus days of the simulation. 

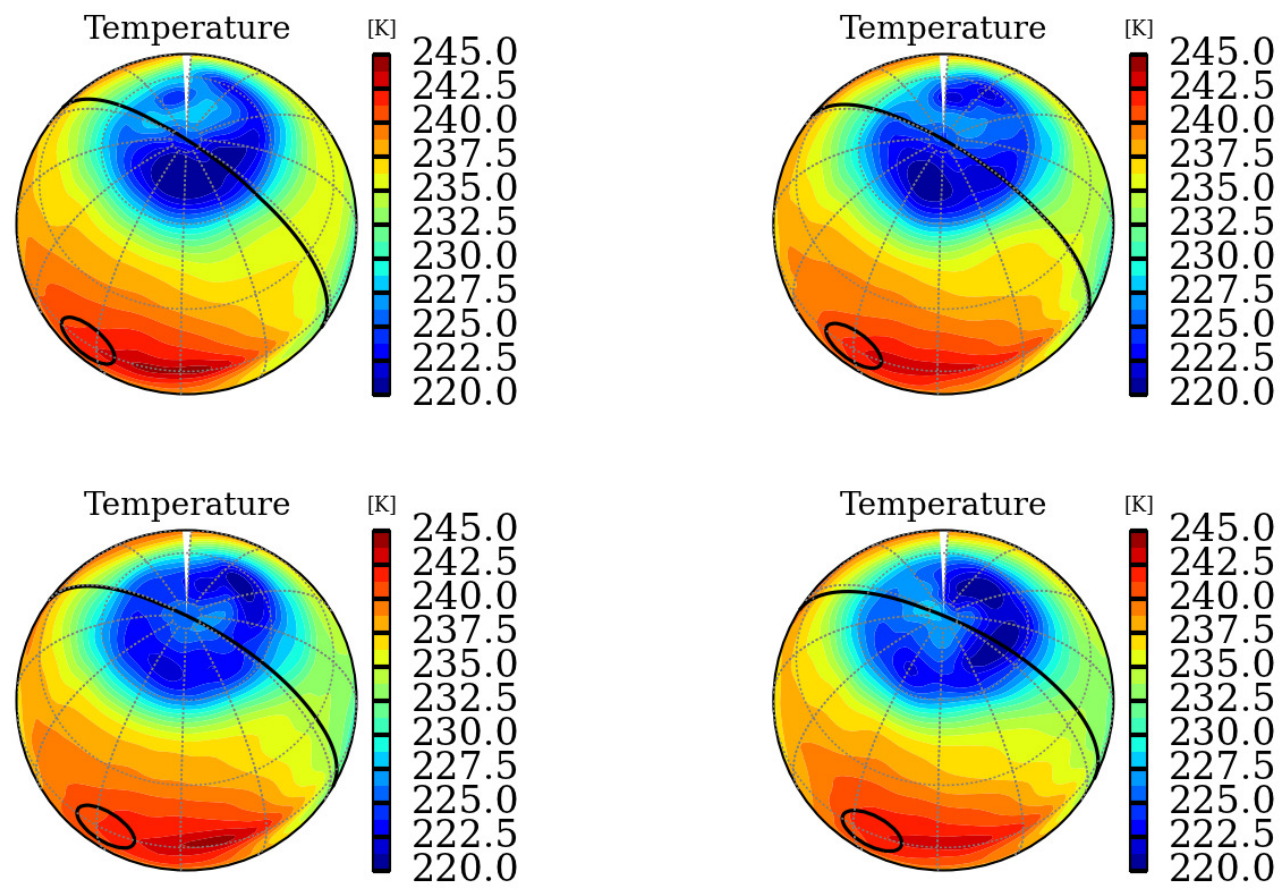

Figure 3: Temperature fields at pressure $\mathrm{p}=3 \times 10^{3} \mathrm{~Pa}(\sim 67 \mathrm{~km}$ altitude $)$ in the northern polar region. In each panel, the temperature field is averaged over 1/100 Vd (1.17 Ed), and the panels are separated by this time interval. The black contours show the sub-solar area and the terminator. The latitude and longitude divisions are $30^{\circ}$. 


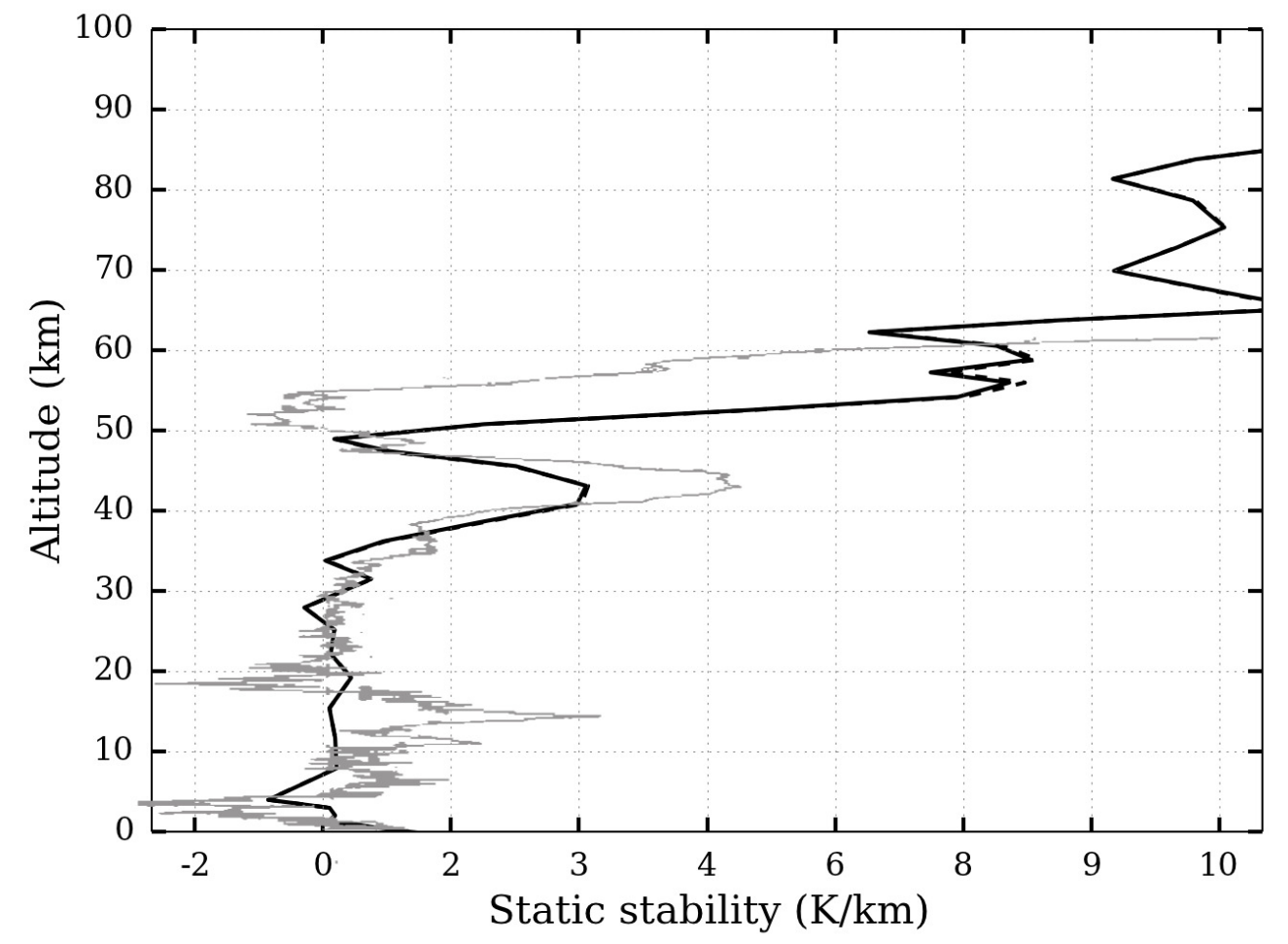

Figure 4: Vertical profile of globally averaged static stability obtained in the simulation started from superrotation, after $190 \mathrm{Vd}$, compared to profile retrieved from the Vega 2 entry probe dataset (adapted from Zasova et al., 2007), shown in gray. The dashed line is from the simulation started from rest, after $300 \mathrm{Vd}$. 


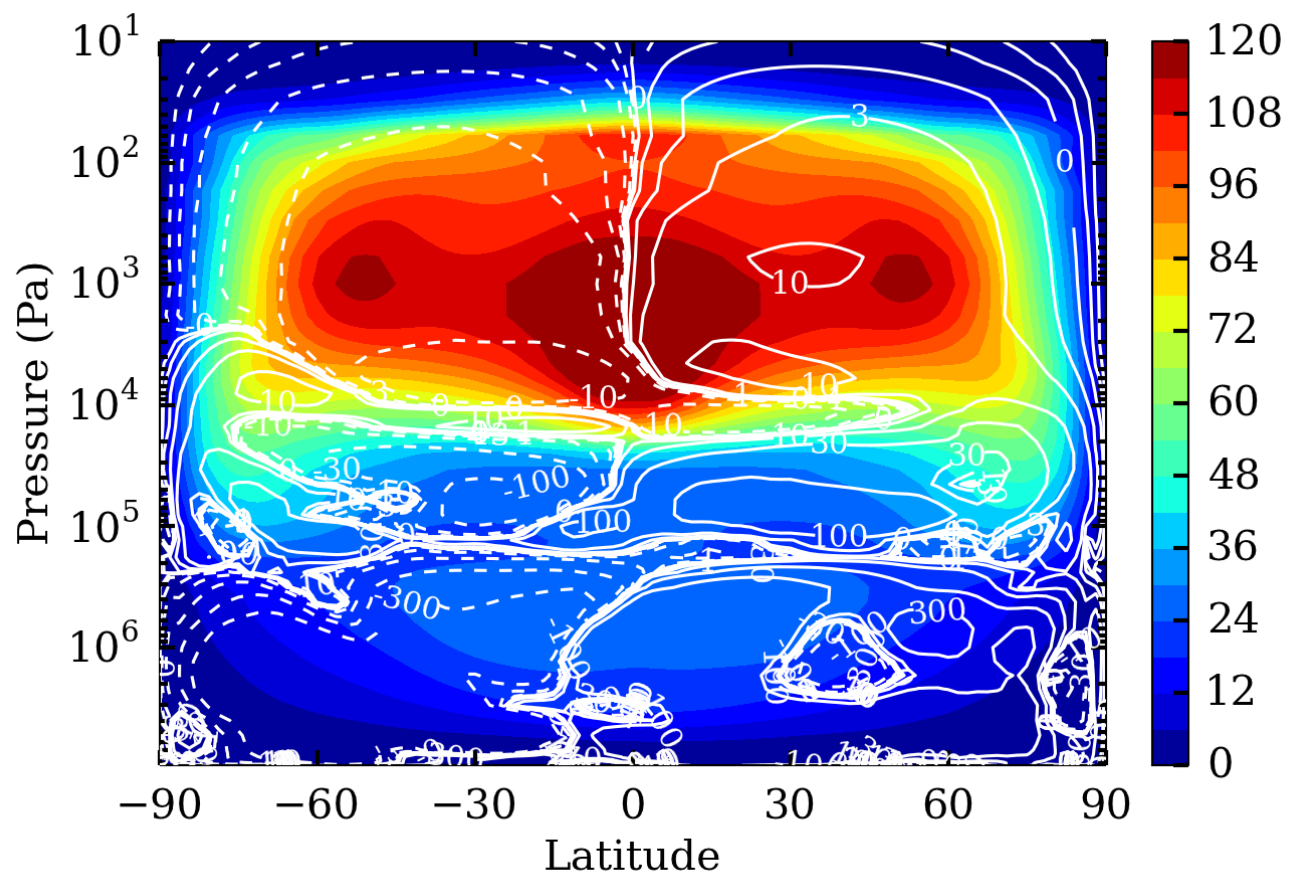

Figure 5: Distribution of the mean zonal wind field (white contours show the mean meridional stream function, in units of $10^{9} \mathrm{~kg} / \mathrm{s}$ ) obtained in the simulation started from superrotation, after 190 Vdays. 
a)

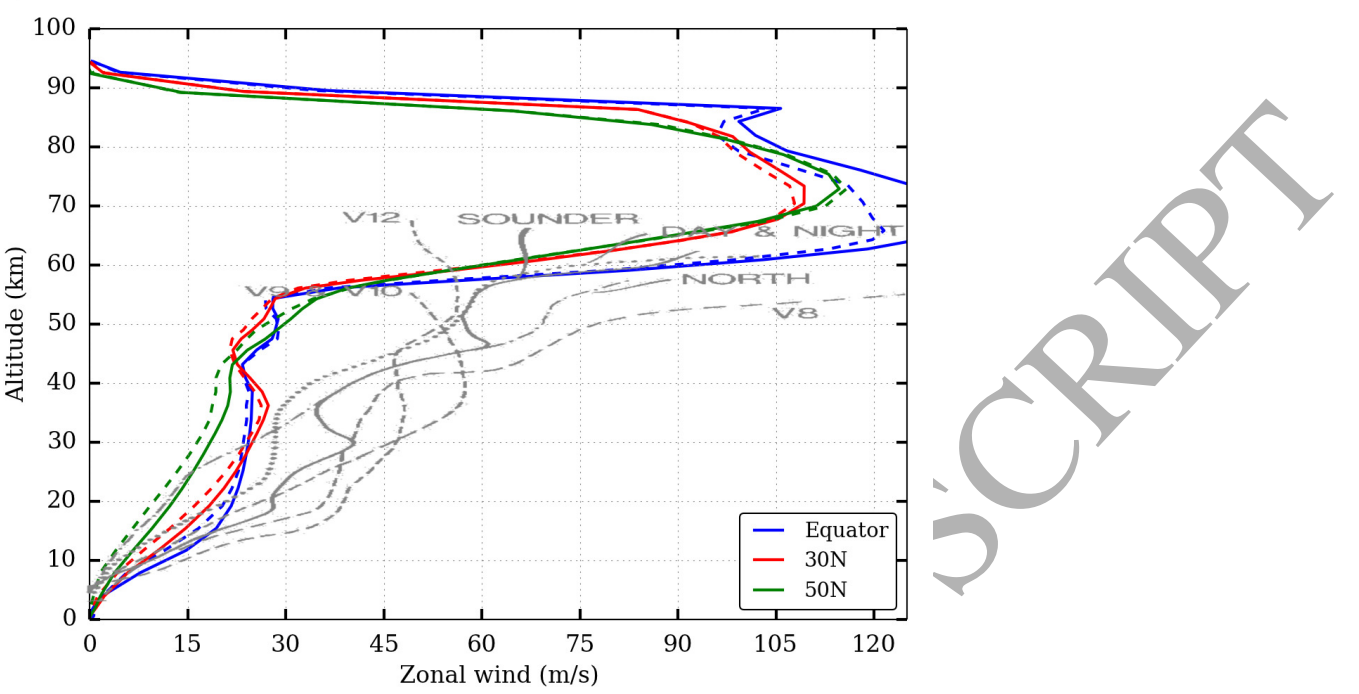

b)

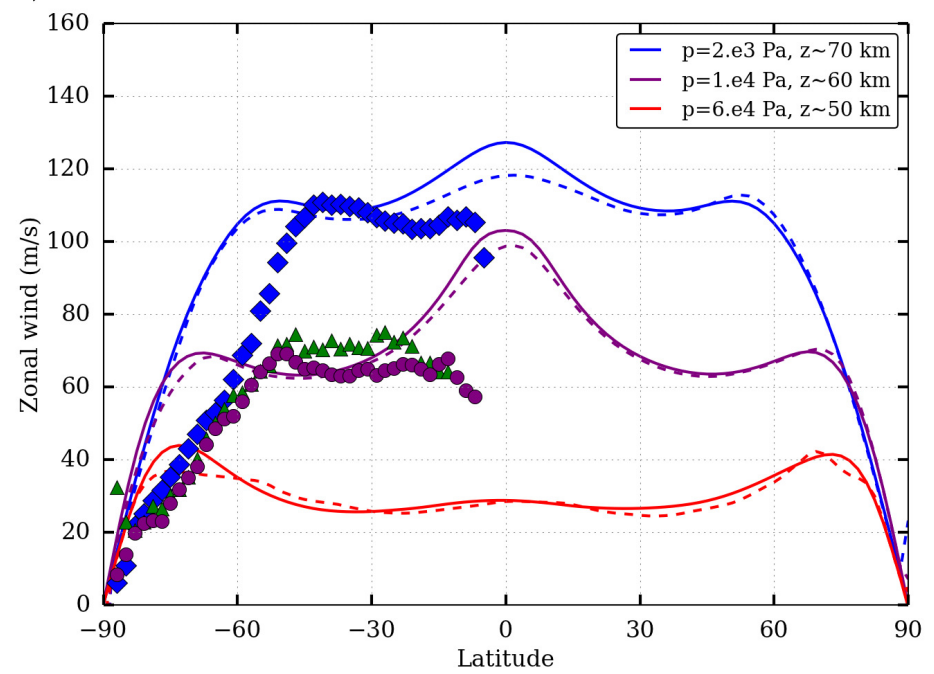

Figure 6: Zonal and temporal averaged profiles of the zonal wind: (a) vertical profiles at three different latitudes, compared to observed profiles from Venera and Pioneer Venus probes (gray, adapted from Schubert, 1983); (b) latitudinal profiles at roughly 50, 60 and $70 \mathrm{~km}$ altitude, compared to averaged cloud-tracking zonal wind profiles obtained with VIRTIS-M images at UV (blue diamonds), visible (green triangles) and near-IR (magenta circles) wavelengths. They correspond to altitudes $66-72 \mathrm{~km}$ for UV spectral range, and a few kilometers below that level for visible/near-IR wavelengths (adapted from Hueso et al., 2015). The dashed lines are from the simulation started from rest, after $300 \mathrm{Vd}$. 
a) Temperature Lat.=Eq.

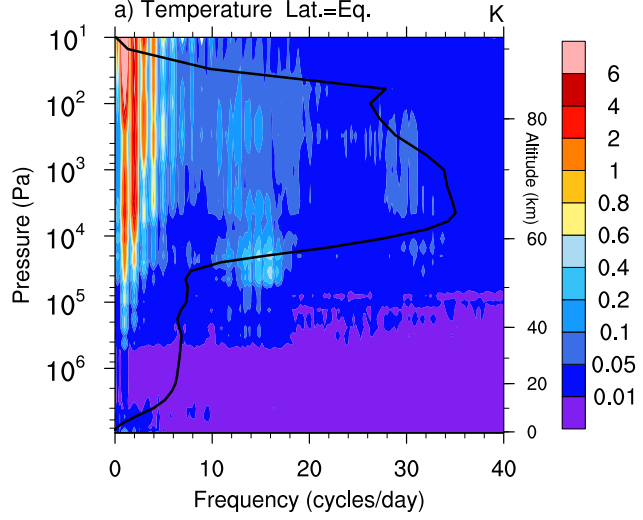

c) Zonal wind Lat.=Eq.

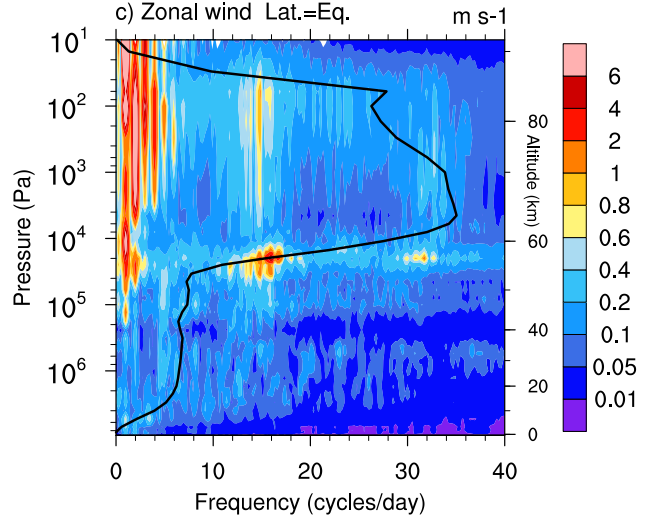

e) Meridional wind Lat.=Eq. $\quad \mathrm{m} \mathrm{s}-1$

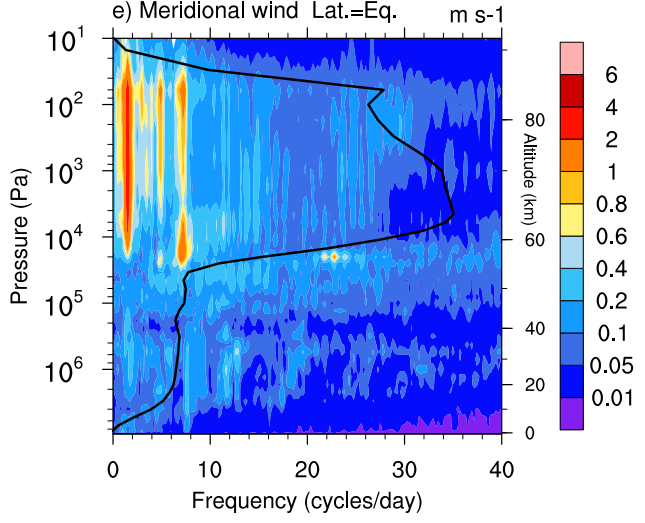

b) Temperature Lat. $=70 \mathrm{~N}$

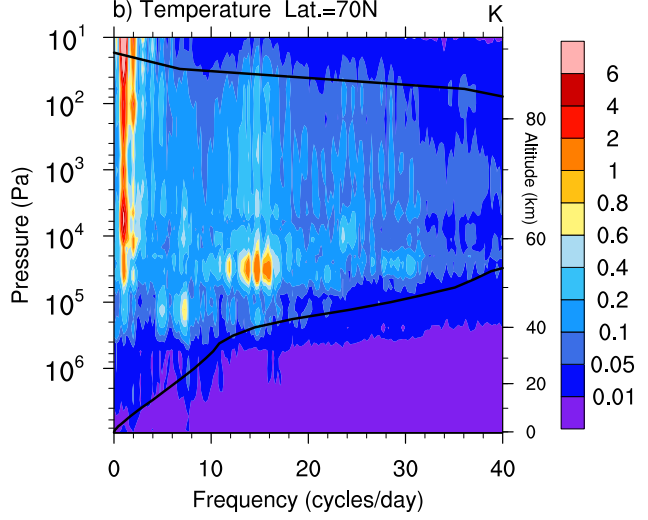

d) Zonal wind Lat. $=70 \mathrm{~N}$
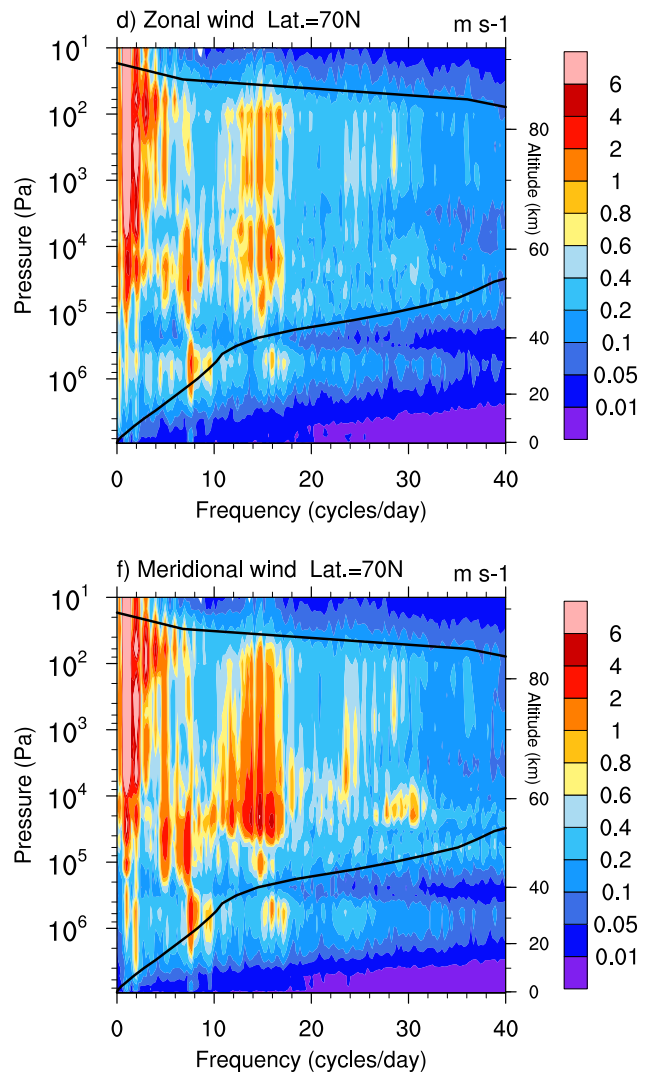

Figure 7: Frequency analysis of the temperature, zonal and meridional wind time series as a function of pressure, at the equator and $70^{\circ} \mathrm{N}$. The solid black line indicates the frequency corresponding to the zonal and temporal average of the zonal wind speed. 

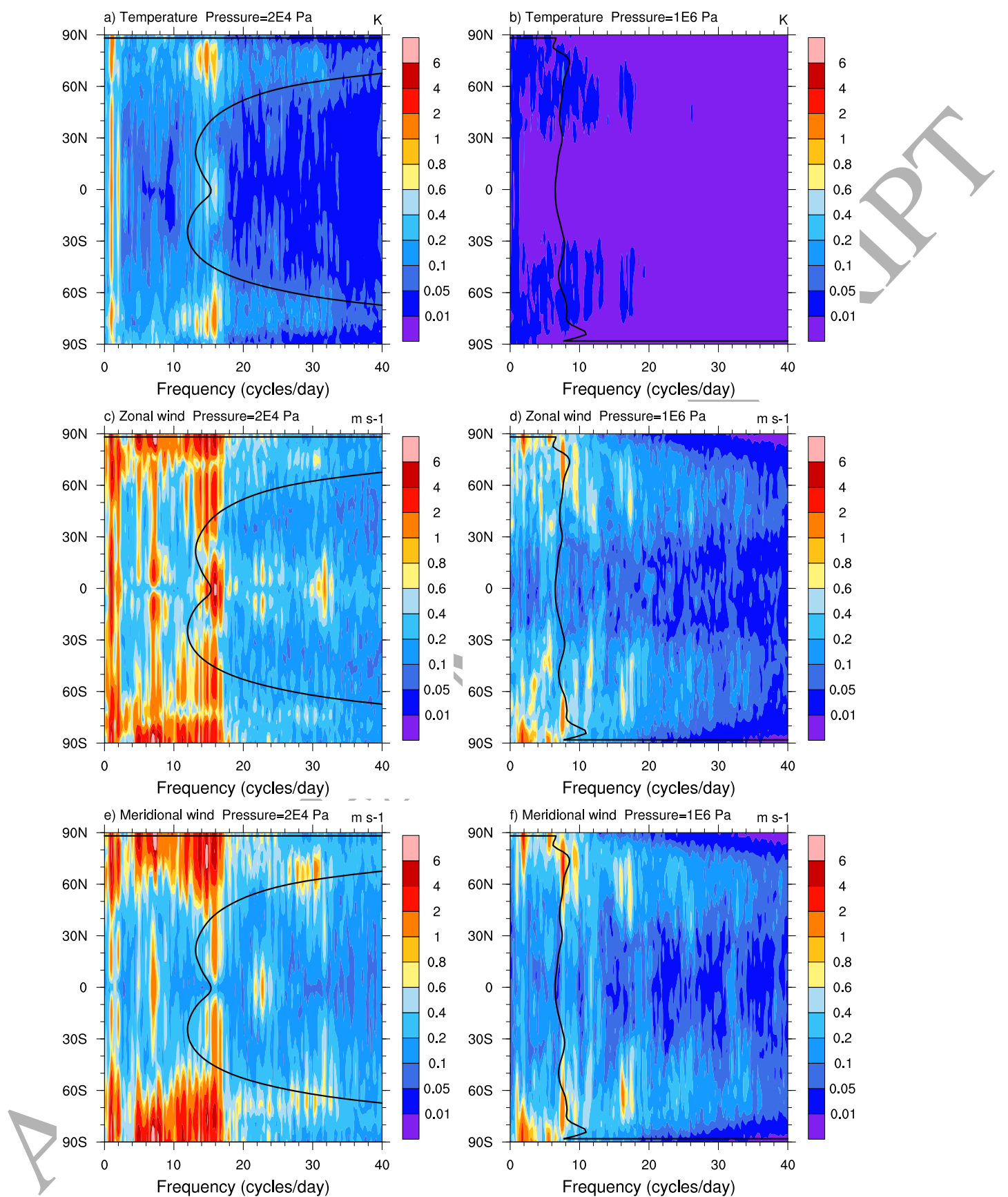

Figure 8: Frequency analysis of the temperature, zonal and meridional wind time series as a function of latitude, at pressure levels $2 \times 10^{4} \mathrm{~Pa}$ and $1 \times 10^{6} \mathrm{~Pa}$. The solid black line indicates the frequency corresponding to thetbonal and temporal average of the zonal wind speed. 


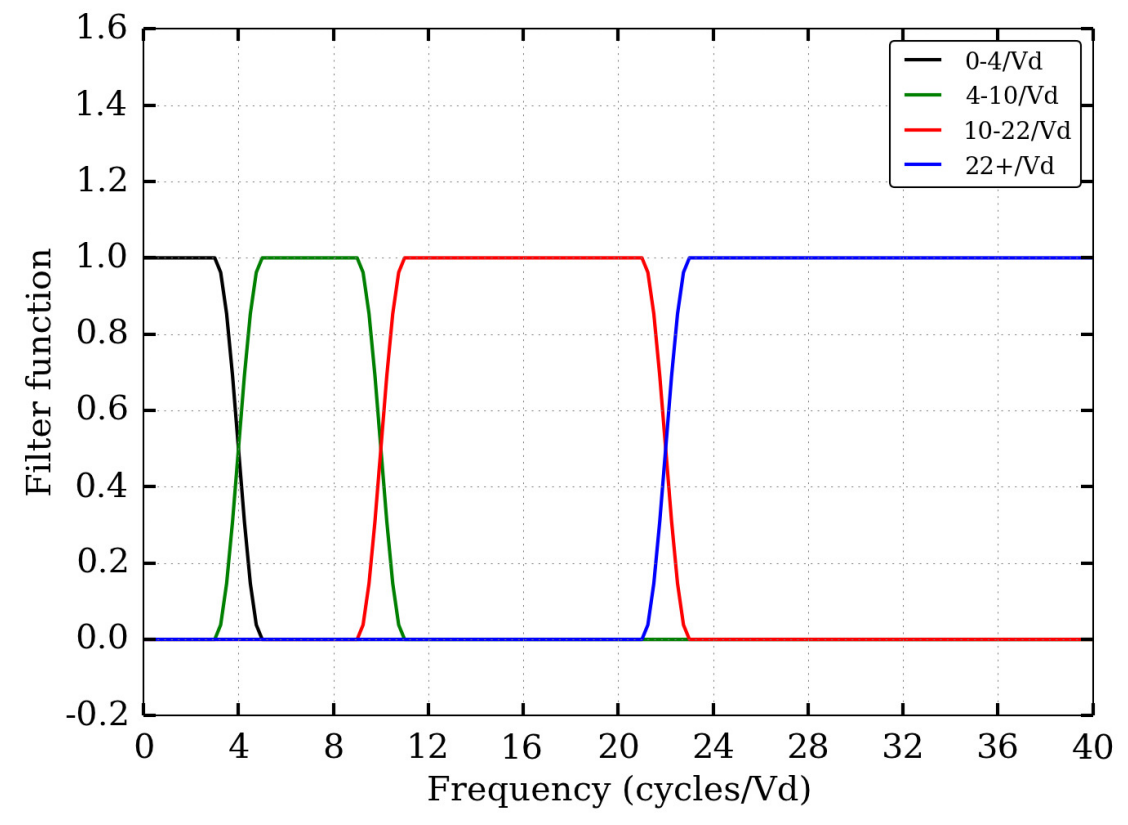

Figure 9: Filter functions applied to the FFT spectra to separate the different waves present in the temperature and wind fields. 


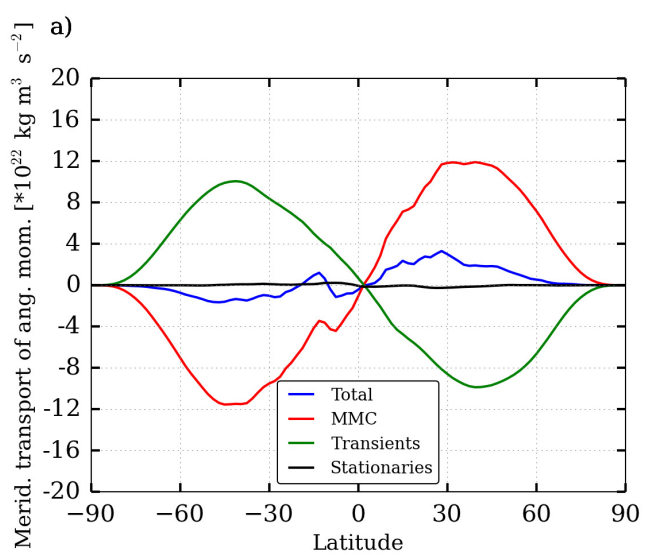

b)

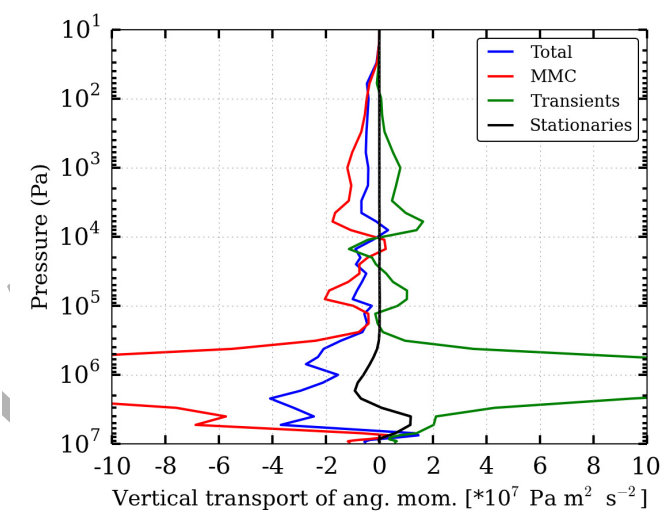

Figure 10: Meridional (a) and vertical (b) transport of angular momentum, separating MMC contribution from transients and stationaries. The net horizontal transport is compensated by exchanges with the solid surface. However, the net vertical transport should be zero and the residual is indicative of the numerical inaccuracies discussed at the end of Section 2.1 .

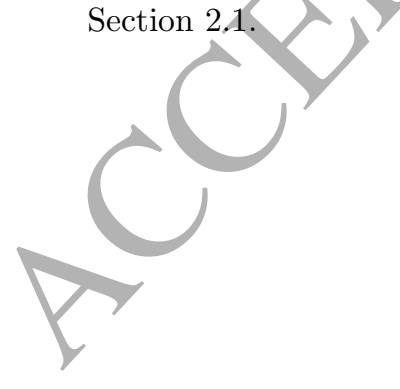



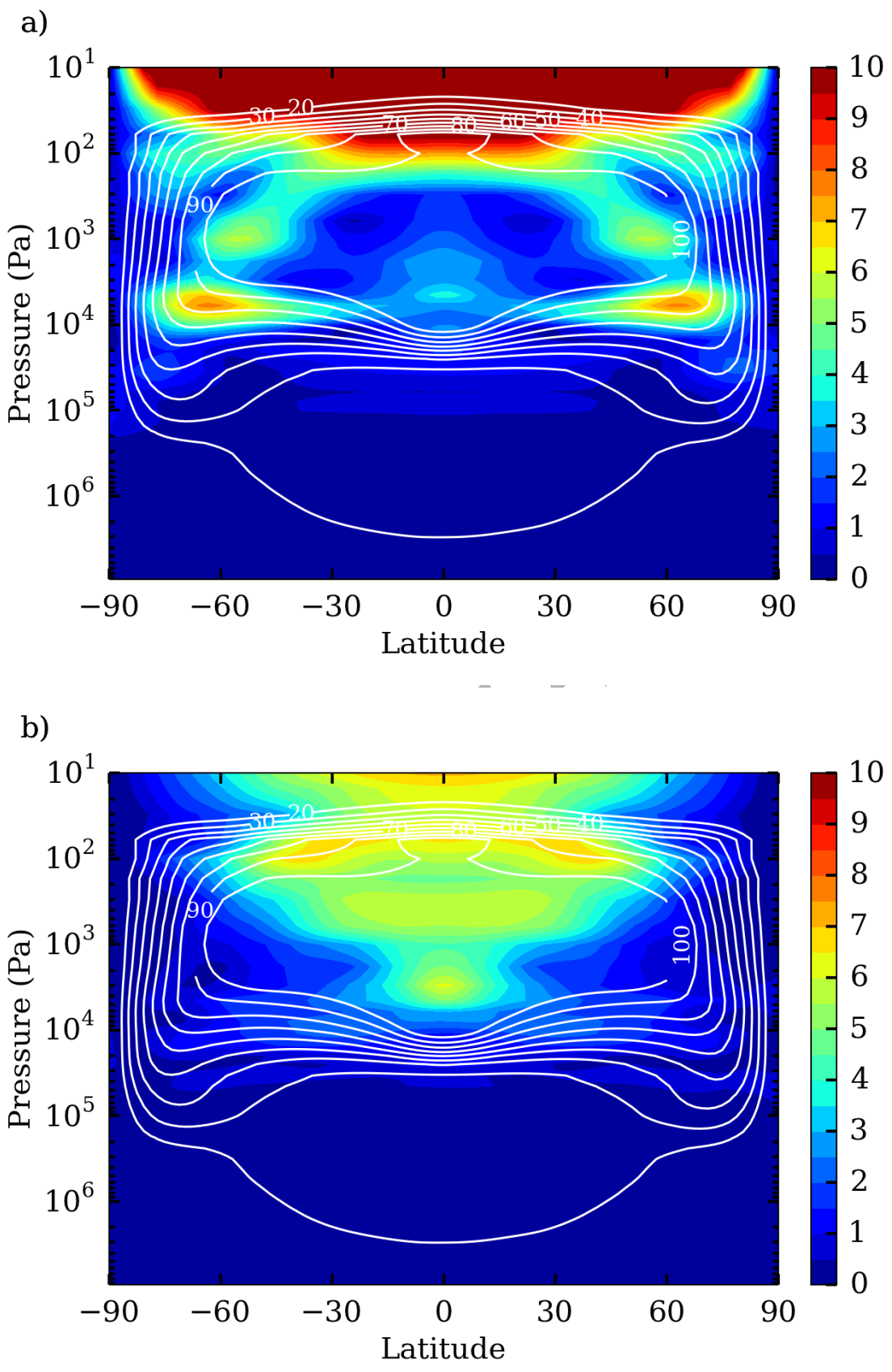

Figure 11: Amplitude of the FFT spectrum of the temperature at the frequency (a) 1/Vd, i.e. diurnal tide and (b) $2 / \mathrm{Vd}$, i.e. semi-diurnal tide. The white contours show the mean zonal wind field (in $\mathrm{m} / \mathrm{s}$ ). 

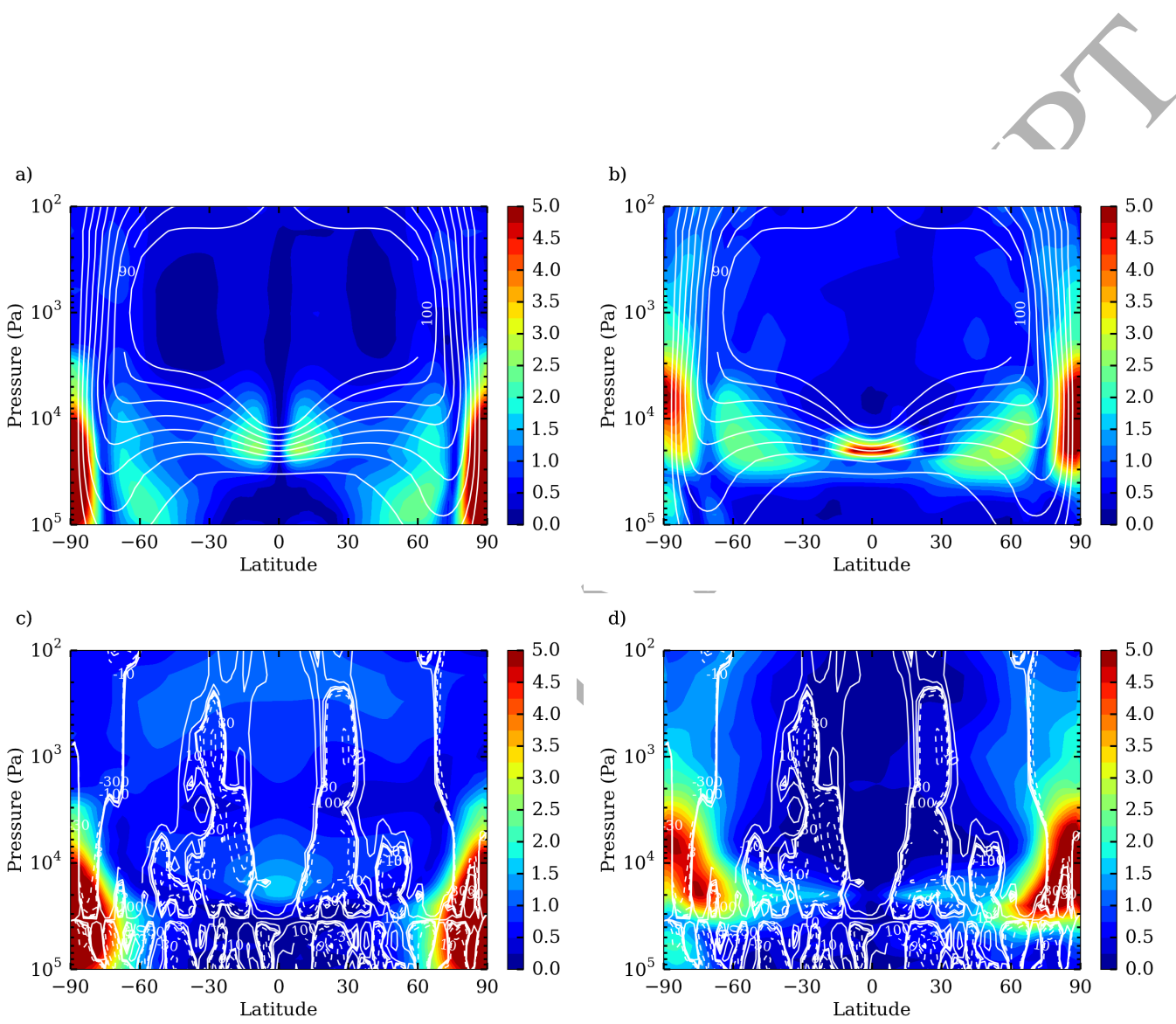

Figure 12: Amplitude of the FFT spectrum of (a,b) the zonal and (c,d) meridional winds, at the frequency $7.25 / \mathrm{Vd}$ (left) and $16 / \mathrm{Vd}$ (right). The white contours show (a,b) the mean zonal wind field (in $\mathrm{m} / \mathrm{s}),(\mathrm{c}, \mathrm{d}) d \bar{q} / d \phi\left(\right.$ in $\left.\mathrm{m} \mathrm{s}^{-1}\right)$. 

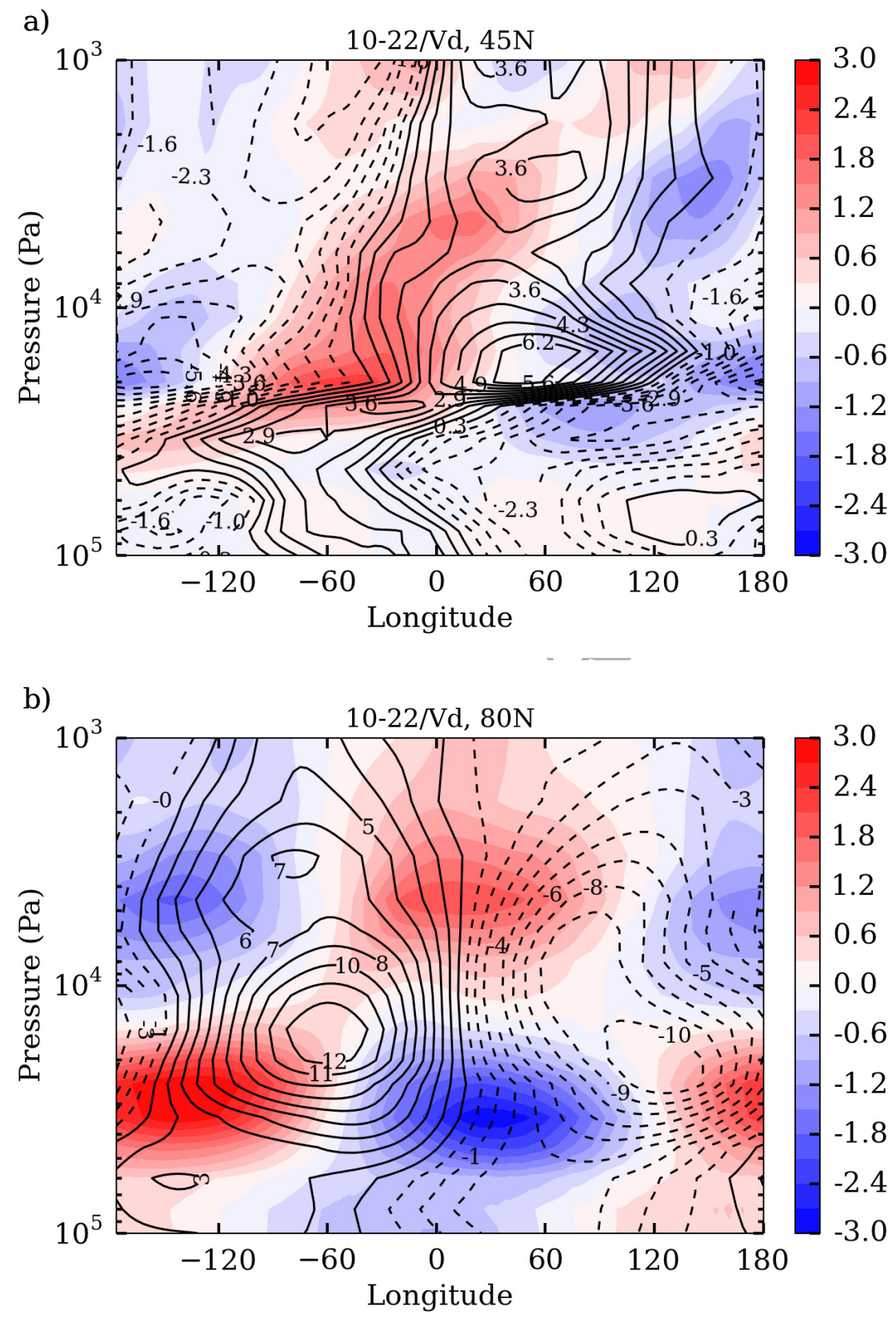

Figure 13: Temperature (colors, in $\mathrm{K}$ ) and meridional wind (contours, in $\mathrm{m} / \mathrm{s}$ ) perturbations filtered in the frequency band $[10-22] / \mathrm{Vd}$, at (a) $45^{\circ} \mathrm{N}$ (baroclinic structures) and (b) $80^{\circ} \mathrm{N}$ (barotropic structures). Note that the zonal wind is moving westward. These perturbations are shown at a fixed point in time (without averaging). 

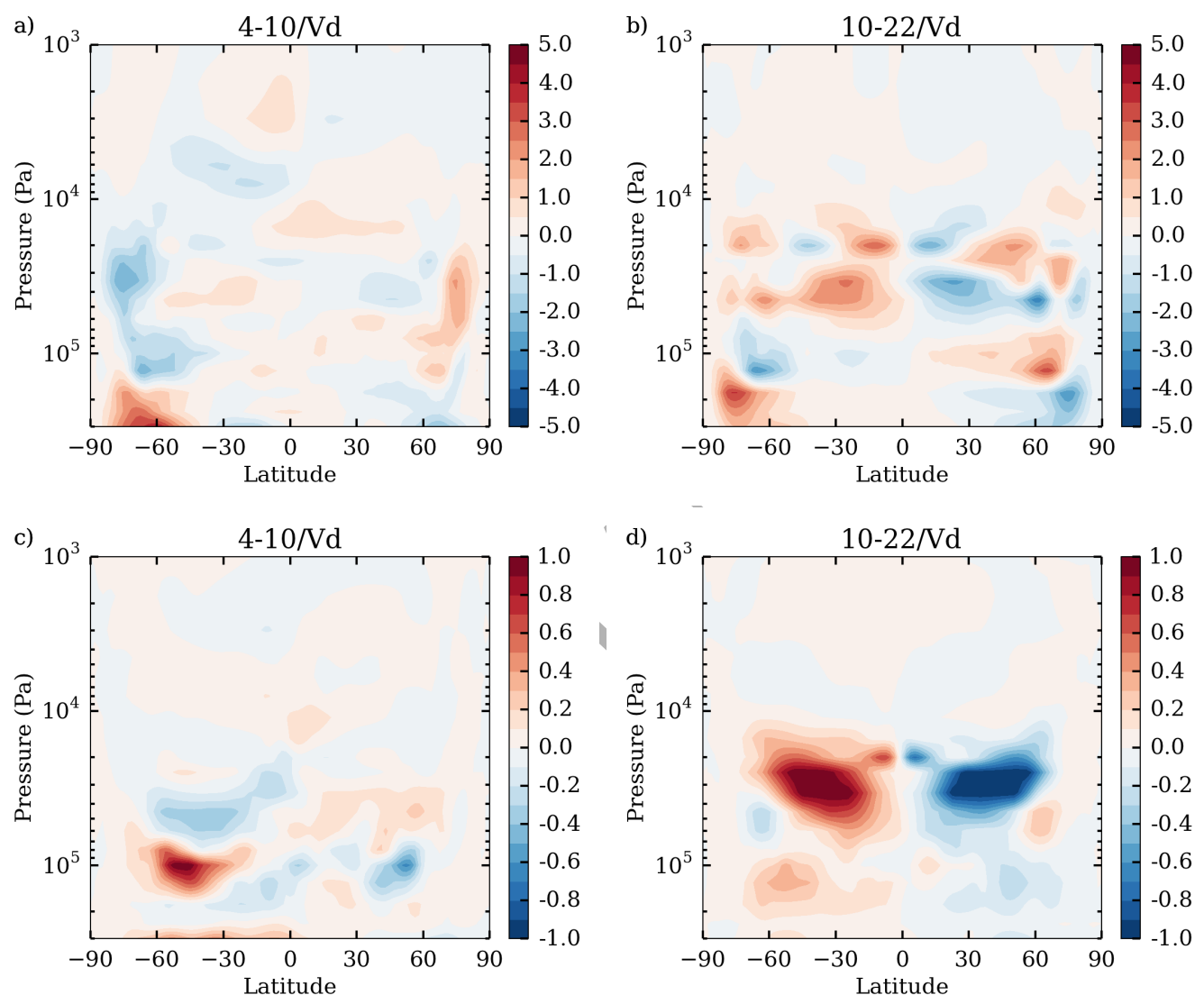

Figure 14: Horizontal transport of heat (upper row) and angular momentum (lower row) computed using zonal and meridional fields filtered in the frequency bands $[04-10] / \mathrm{Vd}$ (left) and [10-22]/Vd (right). Units are $1 \times 10^{16} \mathrm{~J} \mathrm{~m} \mathrm{~s}^{-1}$ for heat transport and $1 \times 10^{21} \mathrm{~kg}$ $\mathrm{m}^{3} \mathrm{~s}^{-2}$ for angular momentum transport. 
a)

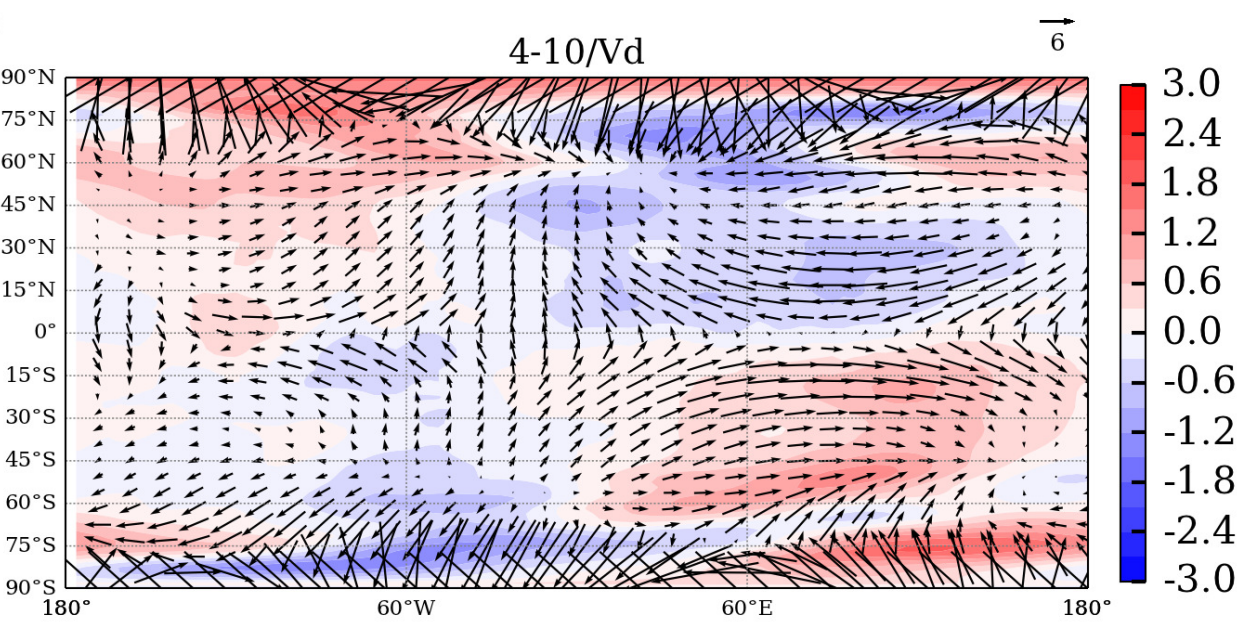

b)

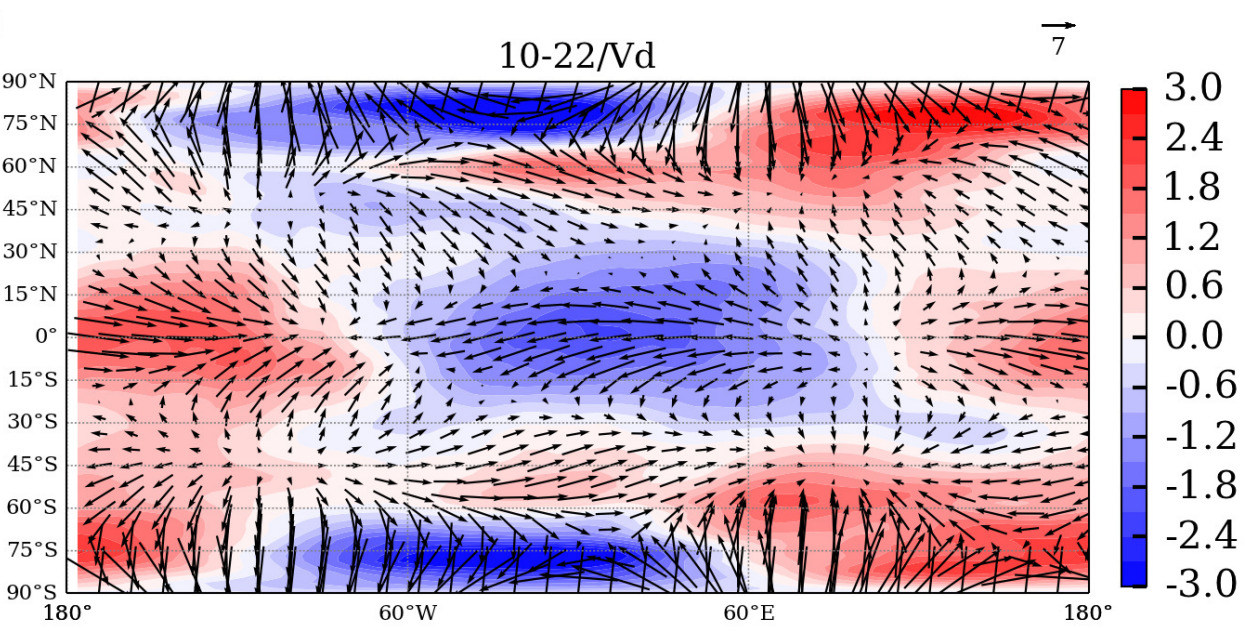

Figure 15: Temperature (colors, in $\mathrm{K}$ ) and horizontal wind (vectors, in $\mathrm{m} / \mathrm{s}$ according to rule) perturbations filtered in the frequency bands (a) [04-10]/Vd and (b) [10-22]/Vd, at $2 \times 10^{4} \mathrm{~Pa}$. These perturbations are shown at a fixed point in time (without averaging). 

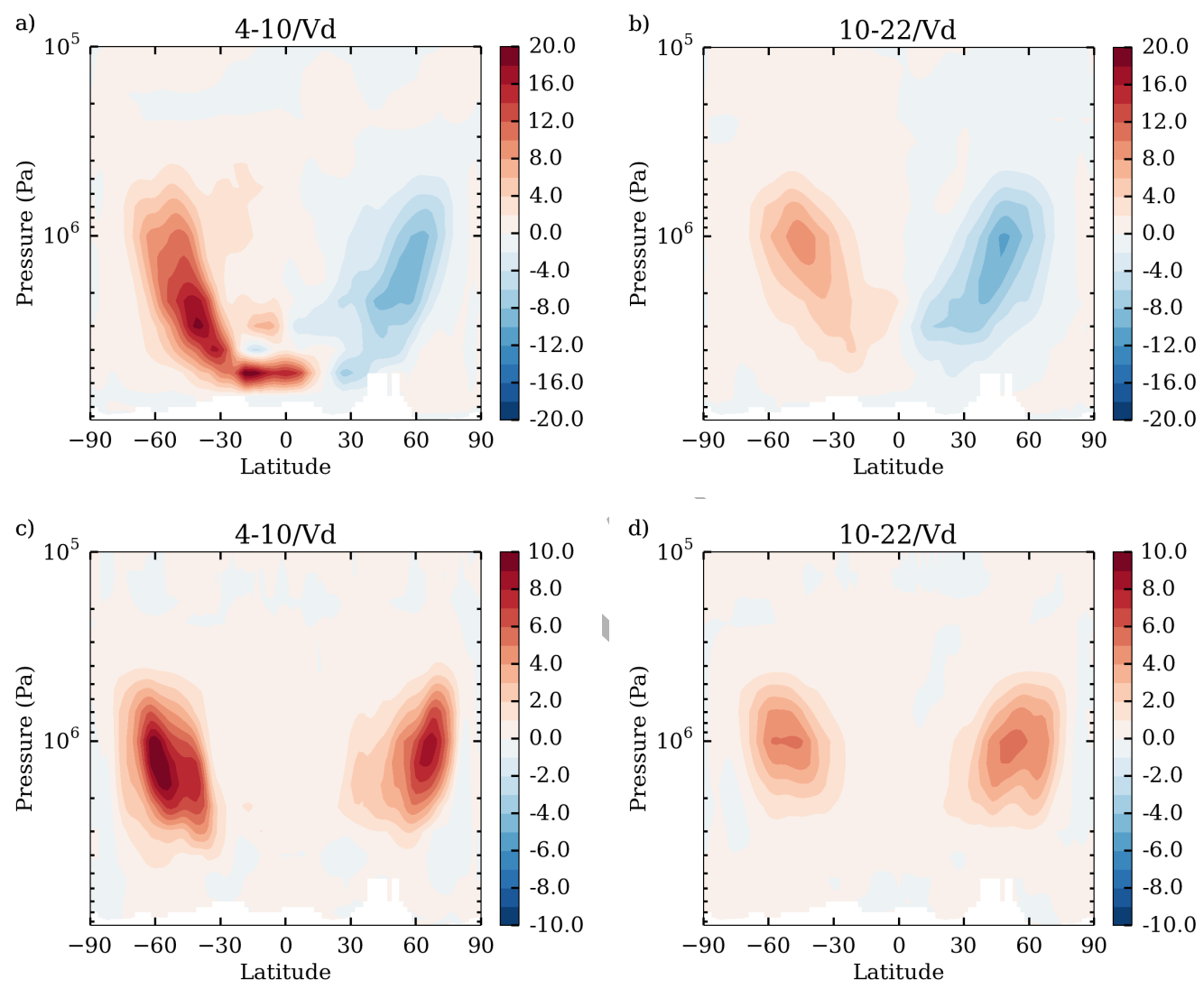

Figure 16: Meridional (top) and vertical (bottom) transport of angular momentum below the clouds computed using zonal and meridional $(\mathrm{a}, \mathrm{b})$ or vertical $(\mathrm{c}, \mathrm{d})$ wind fields filtered in the frequency bands [04-10]/Vd (left) and [10-22]/Vd (right). Units are $1 \times 10^{21} \mathrm{~kg} \mathrm{~m}^{3}$ $\mathrm{s}^{-2}$ for panels a and $\mathrm{b}$ and $1 \times 10^{6} \mathrm{~Pa} \mathrm{~m}^{2} \mathrm{~s}^{-2}$ for panels $\mathrm{c}$ and $\mathrm{d}$. 

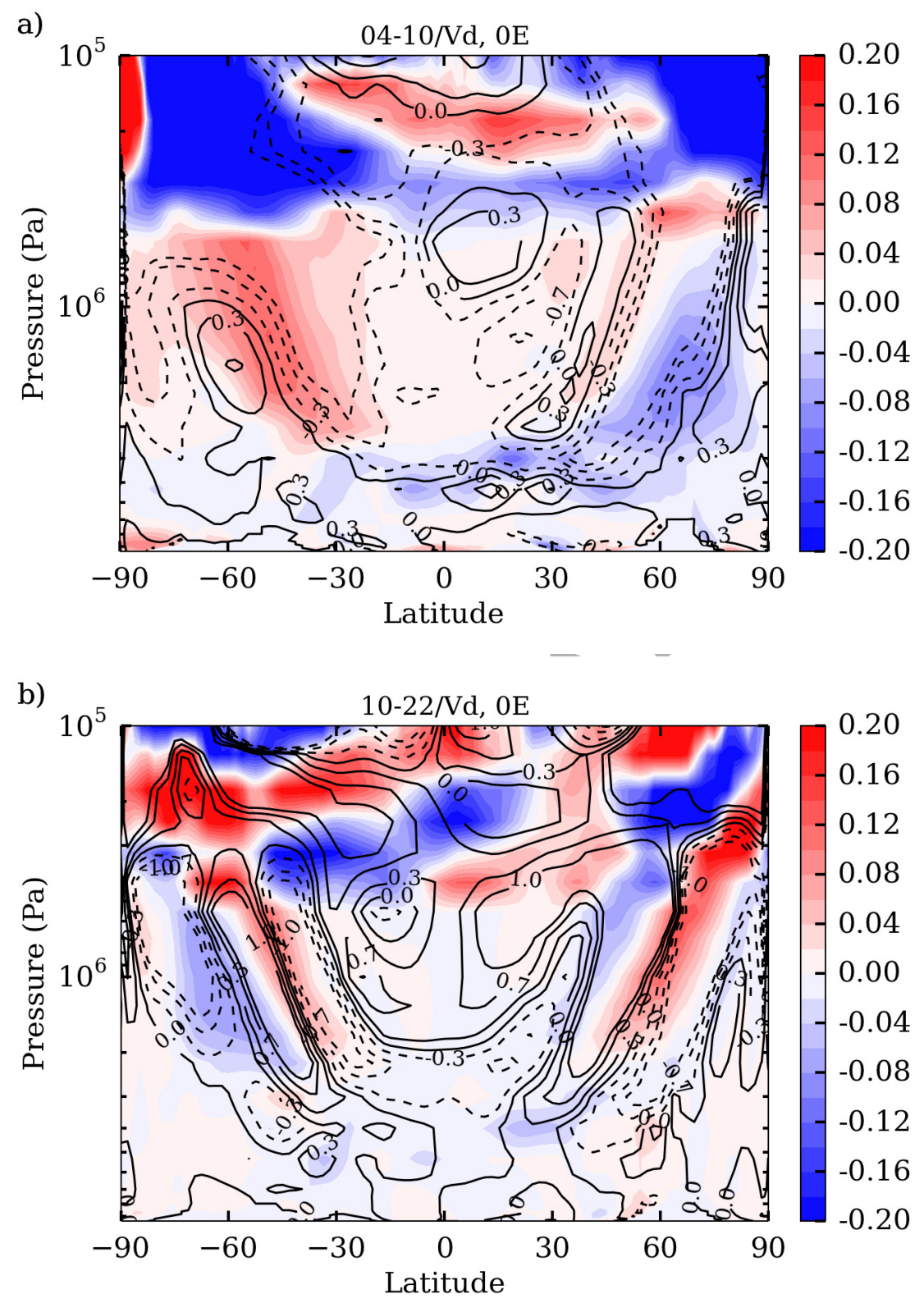

Figure 17: Temperature (colors, in $\mathrm{K}$ ) and meridional wind (contours, in $\mathrm{m} / \mathrm{s}$ ) perturbations filtered in the frequency bands (a) [04-10]/Vd and (b) [10-22]/Vd, at $0^{\circ}$ longitude. These perturbations are shown at a fixed point in time (without averaging). 
a)

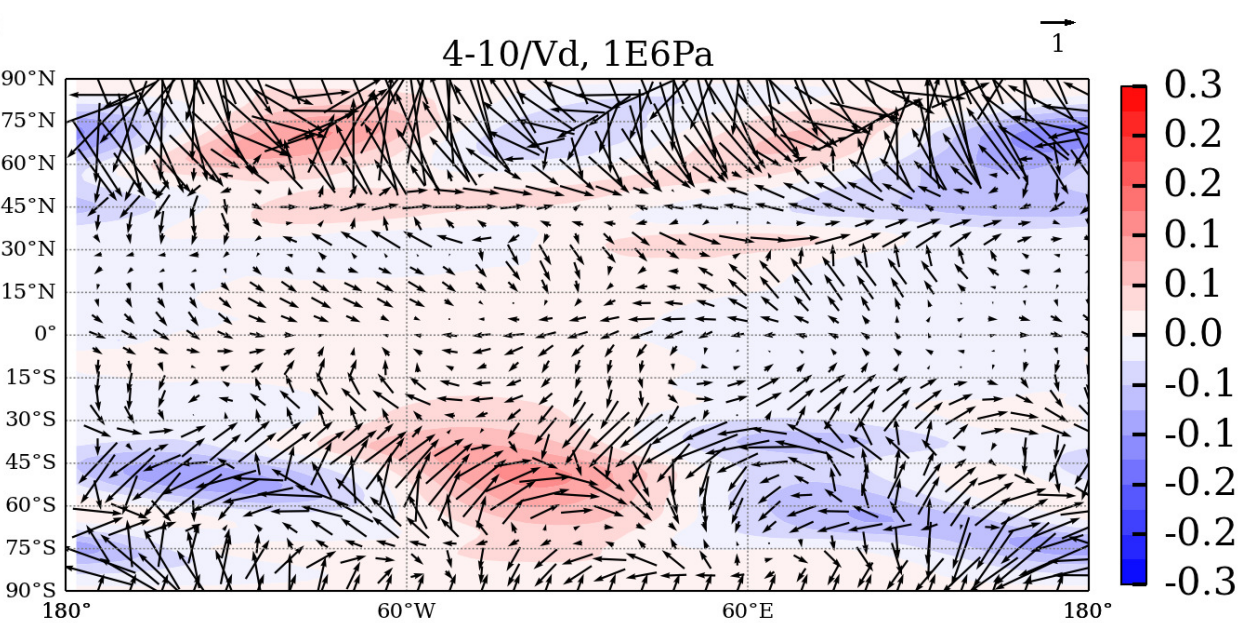

b)

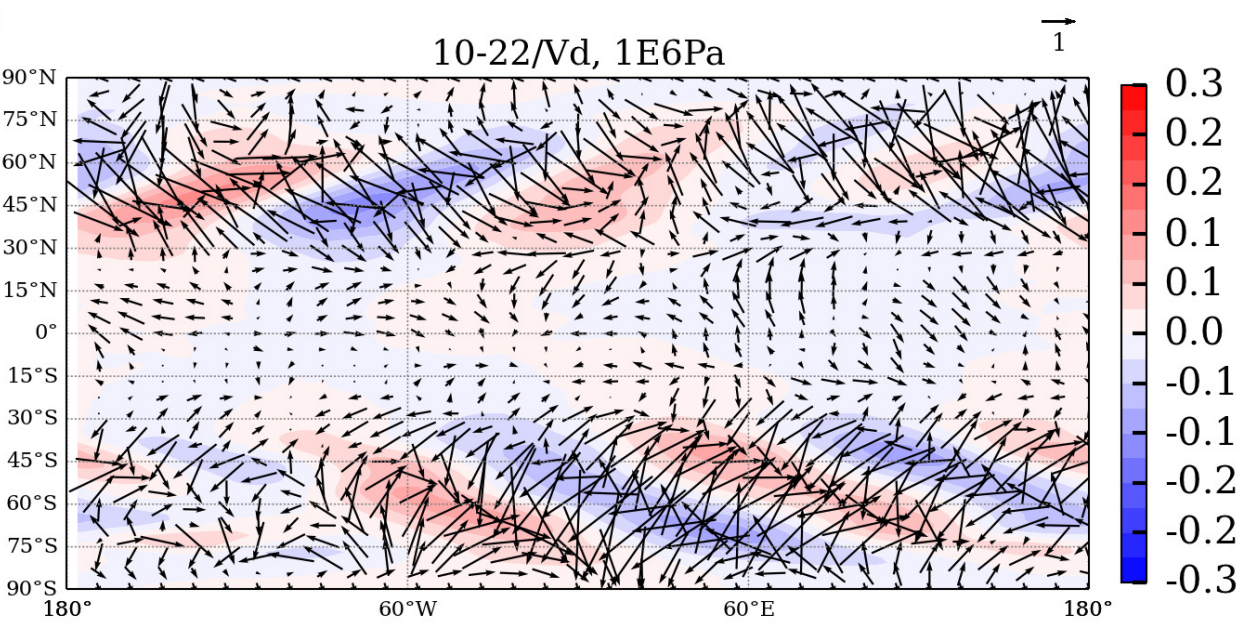

Figure 18: Temperature (colors, in $\mathrm{K}$ ) and horizontal wind (vectors, in $\mathrm{m} / \mathrm{s}$ according to rule) perturbations filtered in the frequency bands (a) [04-10]/Vd and (b) [10-22]/Vd, at $1 \times 10^{6} \mathrm{~Pa}$. These perturbations are shown at a fixed point in time (without averaging). 

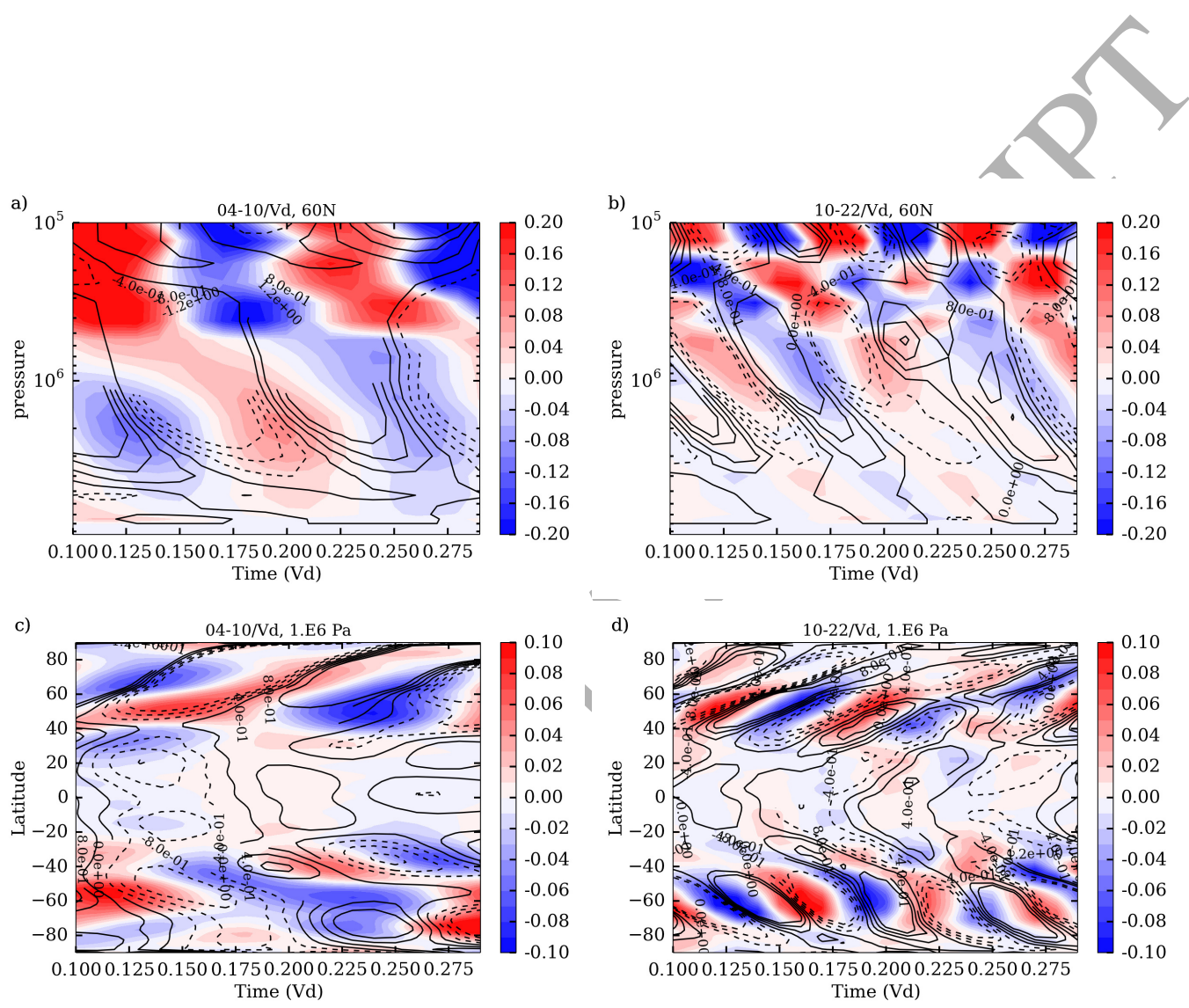

Figure 19: Temporal variations of temperature (colors, in K) and meridional wind (contours, $\mathrm{m} \mathrm{m} / \mathrm{s}$ ) perturbations filtered in the frequency bands [04-10]/Vd (left column) and [10-22] $\mathrm{Vd}$ (xight column), at $0^{\circ}$ longitude, $60^{\circ} \mathrm{N}$ latitude (top) and $1 \times 10^{6} \mathrm{~Pa}$ (bottom).

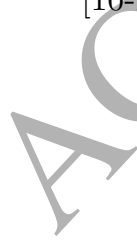

